\title{
Ultrafast Demagnetization of Iron Induced by Optical versus Terahertz Pulses
}

\author{
A. L. Chekhov $\odot,{ }^{1,2}$ Y. Behovits $\odot,{ }^{1,2}$ J. J. F. Heitz ${ }^{1,2}$ C. Denker, ${ }^{3}$ D. A. Reiss, ${ }^{1}$ M. Wolf, ${ }^{2}$ M. Weinelt@, \\ P. W. Brouwer $\odot,{ }^{1}$ M. Münzenberg, ${ }^{3}$ and T. Kampfrath $\oplus^{1,2}$ \\ ${ }^{1}$ Fachbereich Physik, Freie Universität Berlin, Arnimallee 14, 14195 Berlin, Germany \\ ${ }^{2}$ Fritz-Haber-Institut der Max-Planck-Gesellschaft, Faradayweg 4-6, 14195 Berlin, Germany \\ ${ }^{3}$ Institut für Physik, Universität Greifswald, Felix-Hausdorff-Straße 6, 17489 Greifswald, Germany
}

(Received 29 April 2021; revised 15 September 2021; accepted 4 October 2021; published 20 December 2021)

\begin{abstract}
We study ultrafast magnetization quenching of ferromagnetic iron following excitation by an optical versus a terahertz pump pulse. While the optical pump (photon energy of $3.1 \mathrm{eV}$ ) induces a strongly nonthermal electron distribution, terahertz excitation $(4.1 \mathrm{meV})$ results in a quasithermal perturbation of the electron population. The pump-induced spin and electron dynamics are interrogated by the magneto-optic Kerr effect (MOKE). A deconvolution procedure allows us to push the time resolution down to $130 \mathrm{fs}$, even though the driving terahertz pulse is about $500 \mathrm{fs}$ long. Remarkably, the MOKE signals exhibit an almost identical time evolution for both optical and terahertz pump pulses, despite the 3 orders of magnitude different number of excited electrons. We are able to quantitatively explain our results using a nonthermal model based on quasielastic spin-flip scattering. It shows that, in the small-perturbation limit, the rate of demagnetization of a metallic ferromagnet is proportional to the excess energy of the electrons, independent of the precise shape of their distribution. Our results reveal that, for simple metallic ferromagnets, the dynamics of ultrafast demagnetization and of the closely related terahertz spin transport do not depend on the pump photon energy.
\end{abstract}

DOI: 10.1103/PhysRevX.11.041055

\section{INTRODUCTION}

To push writing of magnetic information to ultrafast timescales, it is essential to understand the response of magnetically ordered solids to a quasi-instantaneous perturbation $[1,2]$. A model experiment is the excitation of a ferromagnet by a femtosecond laser pulse [Fig. 1(a)] [3-5]. In ferromagnetic metals like $\mathrm{Fe}, \mathrm{Co}$, and $\mathrm{Ni}$, the resulting ultrafast demagnetization (UDM) is known to proceed on a timescale of $100 \mathrm{fs}$ and yields insights into fundamental material parameters such as the electron-spin equilibration time. In terms of applications, UDM is an important process in all-optical magnetization switching [2]. It is driven by the same force as ultrafast spin transport [6-9] and, thus, relevant for applications such as generation of spin torque [10] and terahertz $(\mathrm{THz})$ electromagnetic pulses [11-13].

An open question regarding UDM is how strongly the magnetization dynamics depends on the nature of the pump-induced electron distribution. In thermal equilibrium, the electron distribution is Fermi-Dirac-like [see

Published by the American Physical Society under the terms of the Creative Commons Attribution 4.0 International license. Further distribution of this work must maintain attribution to the author(s) and the published article's title, journal citation, and DOI. Open access publication funded by the Max Planck Society.
Subject Areas: Magnetism, Optics, Spintronics

Fig. 1(b)]. It becomes strongly nonthermal [14-19] by excitation with an optical pulse, as schematically shown in Fig. 1(b) for a pump photon energy of $3.1 \mathrm{eV}$. On a timescale of approximately $100 \mathrm{fs}$, electron scattering (in particular, with other electrons) again leads to a FermiDirac (thermal) distribution with elevated electron temperature $T_{e}$ [20-22].

Several works emphasize that the initial nonthermal electron distribution can lead to an enhanced quenching rate of the magnetization [16,23,24], for instance, through Bloch states with large spin-orbit coupling [16], so-called hot spots. Other works, however, argue that the nonthermal character of the initial electron distribution is rather negligible in terms of UDM $[5,25,26]$.

To study the impact of the precise shape of the initial pump-induced electron distribution, we propose to use a pump photon energy either much larger $(3.1 \mathrm{eV})$ or smaller (4.1 meV) than the characteristic thermal energy of $k_{B} T_{0}=25 \mathrm{meV}$, where $k_{B}$ is the Boltzmann constant and $T_{0}=300 \mathrm{~K}$ is the equilibrium sample temperature under ambient conditions [Fig. 1(b)]. Straightforward model calculations (see Appendix C) show that this approach allows us to tune the character of the electron distribution right after excitation from highly nonthermal [blue curve in Fig. 1(b)] to Fermi-Dirac-like [black curve in Fig. 1(b)]. It is remarkable that, initially and for the same deposited energy density, the THz pump yields 3 orders of magnitude more excited electrons than the optical pump. 
(a)

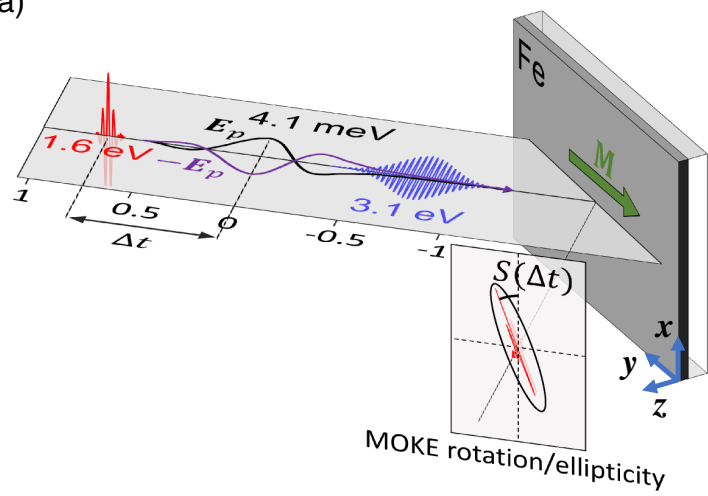

(b)

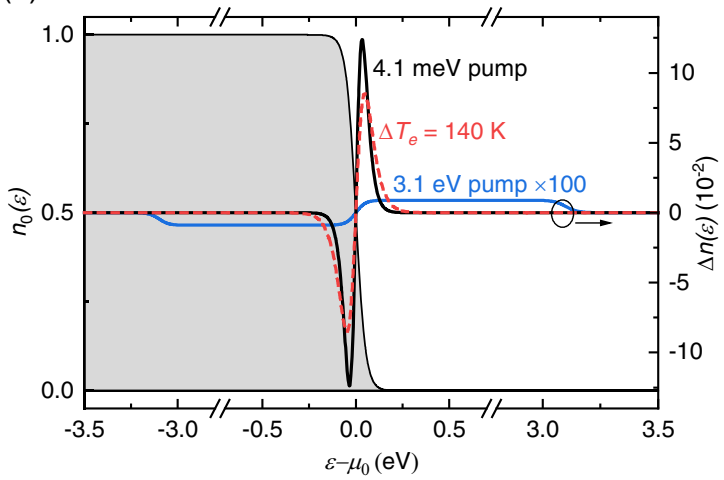

FIG. 1. (a) Schematic of the experiment. A pump pulse at optical (photon energy of $3.1 \mathrm{eV}$ ) or THz $(4.1 \mathrm{meV})$ frequency with transient electric field $\boldsymbol{E}_{p}(t)$ excites a ferromagnetic Fe thin film with in-plane magnetization $\boldsymbol{M}_{0}$ along the $y$ axis. The subsequent sample evolution is monitored by a time-delayed probe pulse $(1.6 \mathrm{eV})$, whose polarization state is detected after reflection from the sample. (b) Calculated variations $\Delta n(\epsilon)$ of the electron occupation number versus electron energy $\epsilon$ after deposition of $0.01 \mathrm{~mJ} / \mathrm{cm}^{2}$ by photons with $4.1 \mathrm{meV}$ (black curve) and $3.1 \mathrm{eV}$ energy (blue curve). We assume constant electronic density of states and transition matrix elements [see Eq. (C29) in Appendix C]. For the THz pump, $\Delta n(\epsilon)$ can be well fit by the difference of two Fermi-Dirac distributions with temperatures $T_{0}+140 \mathrm{~K}$ and $T_{0}=300 \mathrm{~K}$ and the same chemical potential $\mu_{0}$ (red curve). The shaded area shows the unperturbed Fermi-Dirac distribution $n_{0}(\epsilon)$ with temperature $T_{e}=T_{0}=300 \mathrm{~K}$ and chemical potential $\mu_{0}$.

These two distributions imply a very different phase space for subsequent electron scattering and, thus, potentially different magnetization dynamics.

Previous works have already shown that UDM occurs at pump photon energies below $1.5 \mathrm{eV}$, from the infrared $[27,28]$ down to the THz range [29-33]. As the THz pulses have a center frequency of typically $1 \mathrm{THz}$, it is, however, challenging to push the time resolution to the characteristic time constants of electron-spin (approximately $100 \mathrm{fs}$ ) [4,7], electron-electron (approximately 100 fs) [22,34], and electron-phonon equilibration (approximately $500 \mathrm{fs}$ ) [4].

In this work, we measure the ultrafast magnetization dynamics of the model ferromagnet iron (Fe) following ultrashort $\mathrm{THz}(4.1 \mathrm{meV})$ versus optical $(3.1 \mathrm{eV})$ excitation. To directly compare the two datasets, we stay in the linearwith-fluence regime and apply a deconvolution procedure, thereby pushing the time resolution down to $130 \mathrm{fs}$, even though the driving $\mathrm{THz}$ pulse has a duration of $500 \mathrm{fs}$. Surprisingly, the spin dynamics are found to proceed almost identically for both $\mathrm{THz}$ (thermal) and optical (nonthermal) sample excitation. Therefore, the evolution of UDM is predominantly determined by just the amount of deposited pump energy rather than the precise shape of the pump-induced electron distribution. We explain this behavior by an analytical nonthermal model based on quasielastic spin-flip scattering.

\section{EXPERIMENTAL SETUP}

\section{A. Pump-probe experiment}

This section provides the essential features of the experimental setup, and more details can be found in
Appendix A. A schematic of our pump-probe experiment is displayed in Fig. 1(a), and details are shown in Fig. S1(a) [35]. A ferromagnetic Fe thin film is excited by either an optical or a THz pump pulse under an angle of incidence of $45^{\circ}$. The resulting evolution of the electron spin and orbital degrees of freedom is monitored by a time-delayed nearinfrared probe pulse, whose polarization state after reflection off the sample is measured.

As a sample, we choose a ferromagnetic Fe thin film on a glass substrate and capped with $3 \mathrm{~nm} \mathrm{SiO}$. The Fe-layer thickness of $4 \mathrm{~nm}$ is significantly smaller than the attenuation length of the pump (approximately $80 \mathrm{~nm}$ at $1 \mathrm{THz}$ and about $10 \mathrm{~nm}$ at $3.1 \mathrm{eV}$ ) and the probe (approximately $20 \mathrm{~nm}$ ) [36]. Together with the insulating substrate, this condition prevents transport due to field gradients along the sample normal. The sample is characterized with $\mathrm{THz}$ transmission spectroscopy (Fig. S5 [35]) and yields an electron-velocity relaxation time of approximately 8 fs. When the pump is off, the sample magnetization $\boldsymbol{M}_{0}=$ $M_{0} \boldsymbol{u}_{y}$ is in the plane and parallel to the $y$ axis with unit vector $\boldsymbol{u}_{y}$ [Fig. 1(a)].

In our setup, all laser pulses are obtained from an amplified Ti:sapphire laser system. The THz pump pulses [photon energy $4.1 \mathrm{meV}$, duration $500 \mathrm{fs}$ full width at half maximum (FWHM) of intensity, pulse energy $1 \mu \mathrm{J}$, and repetition rate $1 \mathrm{kHz}$ ] are generated by optical rectification of amplified laser pulses (1.55 eV, $50 \mathrm{fs}, 4 \mathrm{~mJ}$, and $1 \mathrm{kHz})$ in a $\mathrm{LiNbO}_{3}$ crystal $[37,38]$. The transient electric field and amplitude spectrum of the $\mathrm{THz}$ pulse are shown in Fig. 2(a). The optical pump pulses $(3.1 \mathrm{eV}, 200 \mathrm{fs}$, $140 \mathrm{~nJ}$, and $1 \mathrm{kHz}$ ) are obtained by frequency doubling of the amplified pulses, and their intensity profile is shown 

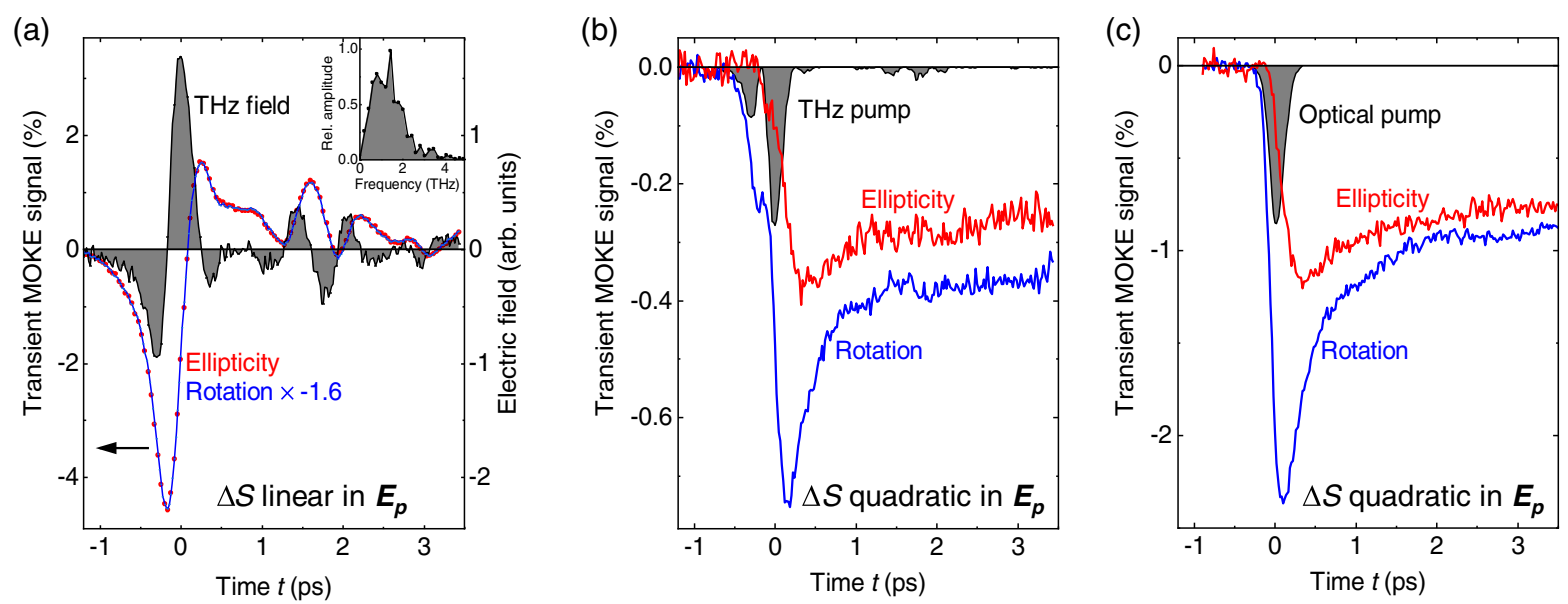

FIG. 2. Typical traces of pump-induced MOKE rotation and ellipticity $\Delta S(t)$ odd in $\boldsymbol{M}_{0}$. (a) Rotation (blue curve, scaled) and ellipticity (red) signals linear in the field $\boldsymbol{E}_{p}$ of the driving THz pump pulse. Assuming this contribution arises from Zeeman torque, its derivative is proportional to the THz magnetic and electric field (black shaded curve). The inset shows the amplitude spectrum of the THz pump field $\boldsymbol{E}_{p}$. (b) THz-pump-induced MOKE rotation (blue) and ellipticity (red) quadratic in $\boldsymbol{E}_{p}$. (c) The same as (b), but for the optical pump pulse. Shaded areas (gray) indicate the corresponding pump intensity profiles $\boldsymbol{E}_{p}^{2}(t)$.

in Fig. 2(c) (gray area). Because the refractive index of Fe at approximately $1 \mathrm{THz}$ is large (about 250) [36], the $\mathrm{THz}$ pump field inside the Fe film is in plane to very good approximation.

As a probe, we use pulses $(1.6 \mathrm{eV}, 40 \mathrm{fs}, 1 \mathrm{~nJ}$, and $80 \mathrm{MHz}$ ) from the seed oscillator of the laser system [Fig. S1(a) [35]] [39,40]. Changes in polarization rotation and ellipticity of the probe upon reflection from the sample are measured by a balanced detection scheme as a function of the delay $t$ since sample excitation by the pump pulse at $t=0$.

\section{B. Expected probe signals}

The detected signal $S(t)$, which equals either the rotation $\left(S^{\text {rot }}\right)$ or ellipticity $\left(S^{\mathrm{ell}}\right)$ of the probe polarization, is up to linear order in the sample magnetization $\boldsymbol{M}$ given by [4143] $S=\boldsymbol{a} \cdot \boldsymbol{M}+\boldsymbol{b}$. The first term is the magneto-optic Kerr effect (MOKE), where the vector $\boldsymbol{a}=\partial S / \partial \boldsymbol{M}$ quantifies how strongly the magnetization affects the linear optical properties of the sample. A nonmagnetic contribution $b$ appears due to a difference between Fresnel reflection coefficients for $s$ and $p$ polarization.

The pump pulse modifies the quantities $\boldsymbol{a}_{0}, \boldsymbol{M}_{0}=M_{0} \boldsymbol{u}_{y}$, and $b_{0}$ of the unperturbed sample by $\Delta \boldsymbol{a}, \Delta \boldsymbol{M}$ and $\Delta b$, respectively. We, thus, measure the pump-induced signal $\Delta S=\Delta(\boldsymbol{a} \cdot \boldsymbol{M}+b)$ as a function of $t$, which equals

$$
\Delta S=\Delta(\boldsymbol{a} \cdot \boldsymbol{M}+b)=\boldsymbol{a}_{0} \cdot \Delta \boldsymbol{M}+M_{0} \Delta a_{y}+\Delta b
$$

up to first order in the pump-induced changes. Note that Eq. (1) applies to both MOKE rotation $S^{\text {rot }}(t)$ and ellipticity $S^{\text {ell }}(t)$. The magneto-optic coupling coefficients are, however, expected to exhibit disparate dynamics [41-44]: $\Delta \boldsymbol{a}^{\mathrm{rot}}(t) \neq \Delta \boldsymbol{a}^{\mathrm{ell}}(t)$.
We expect two contributions to $\Delta \boldsymbol{M}$ : (i) a component $\left.\Delta \boldsymbol{M}\right|_{\mathrm{ZT}}=\Delta M_{z} \boldsymbol{u}_{z}$ perpendicular to the sample plane, which arises from Zeeman-type torque by the magnetic component $\boldsymbol{B}_{p}(t)$ of the pump field [30,45], and (ii) a contribution $\left.\Delta \boldsymbol{M}\right|_{\mathrm{UDM}}=\Delta M_{y} \boldsymbol{u}_{y}$ along the direction of the unperturbed $\boldsymbol{M}_{0}$ due to UDM [29]. To lowest order, contributions (i) and (ii) scale linearly and quadratically in the pump field $\boldsymbol{E}_{p}$, respectively. While component (ii) occurs for both $\mathrm{THz}$ and optical pump, component (i) oscillates rapidly with the optical pump field and, thus, cannot be time resolved with our probe.

To suppress effects of third and higher order in $\boldsymbol{E}_{p}$, we choose the incident pump fluence of the order of $0.1 \mathrm{~mJ} \mathrm{~cm}^{-2}$ (see Fig. S4 [35]). As detailed in Appendix B, we disentangle the various signal contributions by alternating the sign of $\boldsymbol{M}_{0}$ and of the THz pump field $\boldsymbol{E}_{p}$ [33]. First, measuring for $\pm \boldsymbol{M}_{0}$ allows us to separate $\Delta b$ and $\Delta(\boldsymbol{a} \cdot \boldsymbol{M})$ [see Eq. (1)]. Second, alternating the sign of the THz field $\boldsymbol{E}_{p}$ allows us to separate the contributions related to magnetization quenching $\left(\Delta S^{\boldsymbol{M}_{0}, \boldsymbol{E}_{p}^{2}}\right.$ : linear in $\boldsymbol{M}_{0}$ and quadratic in $\boldsymbol{E}_{p}$ ) and Zeeman torque $\left(\Delta S^{\boldsymbol{M}_{0}, \boldsymbol{E}_{p}}\right.$ : linear in both $\boldsymbol{M}_{0}$ and $\left.\boldsymbol{E}_{p}\right)$. Hysteresis-loop measurements of the MOKE signal of the unexcited sample yield $S_{0}\left(\boldsymbol{M}_{0}\right)-S_{0}\left(-\boldsymbol{M}_{0}\right)=2 a_{0 y} M_{0}$, which is used to normalize the pump-probe traces [see Eq. (B2)].

\section{RESULTS}

\section{A. Pump-probe signals}

Figure 2 displays MOKE signals obtained following THz [Figs. 2(a) and 2(b)] and optical pumping [Fig. 2(c)]. The full set of the underlying raw data is shown in Fig. S2 [35]. All signals depend linearly or quadratically on the pump field amplitude (Fig. S4 [35]). 
Figure 2(a) shows signals $\Delta S^{\boldsymbol{M}_{0}, \boldsymbol{E}_{p}}$ following $\mathrm{THz}$ excitation, odd in $\boldsymbol{M}_{0}$ and linear in $\boldsymbol{E}_{p}$. Note that the rotation and ellipticity signal exhibit the same dynamics, which indicates that we observe exclusively true magnetization dynamics here $\left(\Delta a_{y}=0\right)$. Indeed, in electric-dipole approximation, a pump-induced change $\Delta \boldsymbol{a}$ odd in $\boldsymbol{E}_{p}$ is not allowed in the bulk of our nominally inversionsymmetric Fe thin film. A nonvanishing magnetization change, on the other hand, is possible only in magneticdipole approximation and exclusively affects $M_{z}$. This fact is fully consistent with Zeeman torque as the microscopic excitation mechanism, which obeys $\left.\Delta \dot{\boldsymbol{M}}\right|_{\mathrm{ZT}} \propto \boldsymbol{B}_{p}(t) \times \boldsymbol{M}_{0}$. Therefore, by taking the time derivative of the MOKE signals in Fig. 2(a), we consistently obtain a waveform that is proportional to the $\mathrm{THz}$ magnetic and electric field inside the Fe film. The $\mathrm{THz}$ waveforms extracted from the Zeeman-torque transient are in excellent agreement with waveforms obtained by electro-optic sampling in quartz [see Figs. S3(a) and S3(b) [35] and Appendix A].

Figure 2(b) shows MOKE signals $\Delta S^{\boldsymbol{M}_{0}, \boldsymbol{E}_{p}^{2}}$ following $\mathrm{THz}$ excitation, odd in $\boldsymbol{M}_{0}$ and quadratic in $\boldsymbol{E}_{p}$. The signals rise over the duration of the pump pulse and decay subsequently. The double-peak structure of the THz-driven dynamics coincides with the double peak in the $\mathrm{THz}$ intensity profile [shaded area in Fig. 2(b)]. Figure 2(c) displays corresponding signals $\Delta S^{\boldsymbol{M}_{0}, \boldsymbol{E}_{p}^{2}}$ following optical excitation. Analogous to the $\mathrm{THz}$ case, the $\Delta S^{\boldsymbol{M}_{0}, \boldsymbol{E}_{p}^{2}}$ rise with arrival of the pump and decay subsequently.

Note that the rotation and ellipticity signals in Figs. 2(b) and 2(c) exhibit substantially different dynamics. Therefore, the signal quadratic in the pump field $\boldsymbol{E}_{p}$ contains pump-induced variations $\Delta \boldsymbol{a}$ of the magneto-optic coefficients [Eq. (1)] and, thus, cannot be interpreted as pure magnetization dynamics. This point is, however, no issue here, because we are primarily interested in a comparison of $\mathrm{THz}$ and optical pumping. Further below, we approximately extract $\Delta \boldsymbol{M}(t)$ from our data.

\section{B. Deconvoluting the pump profile}

We aim to compare the signals following optical and $\mathrm{THz}$ excitation quadratic in the pump field $\boldsymbol{E}_{p}$. This goal is not straightforward, as the measured signals depend on the shape of the pump pulse, which is very different for optical and THz excitation. As seen in Fig. 2, the intensity trace of the optical pump [Fig. 2(c)] is a single peak (200 fs FWHM), whereas the intensity trace of the THz pump [Fig. 2(b)] has, in addition, a sizable preceding peak (approximately 500 fs FWHM).

To solve this issue, we reshape the signals such that they refer to fictitious optical and $\mathrm{THz}$ pump pulses with the same intensity profile and shorter duration than in the experiment. As the relevant signals [Figs. 2(b) and 2(c)] are quadratic in $\boldsymbol{E}_{p}$, they can be written as a convolution

$$
\Delta S(t)=\left(H * \boldsymbol{E}_{p}^{2}\right)(t)=\int d \tau H(t-\tau) \boldsymbol{E}_{p}^{2}(\tau)
$$

of the pump intensity profile $\boldsymbol{E}_{p}^{2}(t)$ and the intrinsic response $H(t)$ of the system. Equation (2) implies that the two interactions with the pump field $\left[\boldsymbol{E}_{p}\left(\tau_{1}\right)\right.$ and $\left.\boldsymbol{E}_{p}\left(\tau_{2}\right)\right]$ occur with negligible delay $\left(\tau_{2}-\tau_{1}=0\right)$. This assumption is reasonable, because the memory time is given by the dephasing of electronic states at optical frequencies (approximately $20 \mathrm{fs}$ in metals [46]) and the transport relaxation time (approximately 8 fs in our sample; see Fig. S5 [35]) for THz excitation. In Eq. (2), convolution with the probe intensity profile is neglected, because the probe pulse is significantly shorter than the pump.

Deconvolution of Eq. (2) is possible because the $\boldsymbol{E}_{p}^{2}(t)$ are accurately known and have nominally the same shape for each pulse in the train of pump pulses. The procedure is detailed in Appendix B and Fig. S6 [35]. It delivers a signal $\Delta S_{\mathrm{dec}}(t)=\left(H * \boldsymbol{E}_{\mathrm{fic}}^{2}\right)(t)$ that would be obtained for a fictitious pump pulse whose intensity profile $\boldsymbol{E}_{\text {fic }}^{2}(t)$ is shown by the gray area in Fig. 3. Notably, by using this approach, we push the time resolution of all signal traces down to the same value of $130 \mathrm{fs}$, even though the driving $\mathrm{THz}$ pulse is 500 fs wide [Fig. 2(b)]. Because the considered signals are linear in $\boldsymbol{E}_{p}^{2}$, we scale the $\Delta S_{\mathrm{dec}}$ for the THz pump to match the traces for the optical pump at a delay of $t \approx 2 \mathrm{ps,}$ where electron and phonon baths should be almost fully thermalized [4].

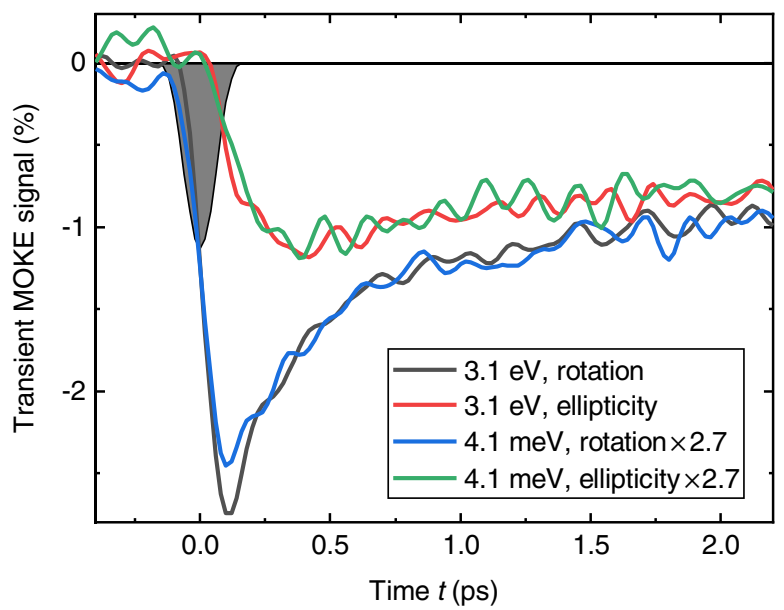

FIG. 3. Pump-induced variations of MOKE rotation and ellipticity $\Delta S^{\boldsymbol{M}_{0}, \boldsymbol{E}_{p}^{2}}(t)$ odd in the sample magnetization $\boldsymbol{M}_{0}$ and quadratic in the pump field $\boldsymbol{E}_{p}$. All signals are deconvoluted with respect to the intensity profile $\boldsymbol{E}_{p}^{2}(t)$ of optical $(3.1 \mathrm{eV})$ and $\mathrm{THz}$ ( $4.1 \mathrm{meV}$ ) pump pulses. They refer to the same fictitious pump profile $\boldsymbol{E}_{\text {fic }}^{2}(t)$ as shown by the shaded black curve. Signals for the THz-pump data are scaled to match the optical one. The scaling factor of 2.7 corresponds to the ratio of the absorbed optical and THz pump fluences (see Appendix A). 
Deconvoluted traces $\Delta S_{\text {dec }}(t)$ for the optical and $\mathrm{THz}$ pump are shown in Fig. 3. The high-frequency oscillations arise from the deconvolution procedure, which amplifies noisy high-frequency components of the measured pumpprobe signal (see Appendix B and Fig. S6 [35]). Figure 3 reveals a remarkable feature: The signals for the optical and $\mathrm{THz}$ pump exhibit very similar dynamics. This finding is consistent with the raw signals in Fig. 2, whose shapes agree quite well, apart from differences during overlap of pump and probe pulses around $t=0$.

In summary, optical and $\mathrm{THz}$ pump pulses are found to induce almost identical dynamics of the MOKE signal [Eq. (1)]. Therefore, the dynamics of the magnetization is almost identical, too. This observation is surprising because (i) the number of initially photoexcited nonthermal electrons differs by 3 orders of magnitude $\left(3.1 \mathrm{eV} / 4 \mathrm{meV} \sim 10^{3}\right)$, and (ii) the distribution $\Delta n(\epsilon, t)$ of those carriers is strongly different (see Fig. 1). Thus, the data in Fig. 3 indicate that thermalization of electrons and their exact distribution after excitation do not play a dominant role in UDM on the scale of our time resolution of $130 \mathrm{fs}$.

\section{Modeling UDM}

To understand this observation, we follow Ref. [47] and model UDM by spin-flip scattering of electrons in a Stonertype approach to ferromagnetism. As indicated in Fig. 4(a) and detailed in Appendix C, the dominant spin-flip events are assumed to arise from impurities and phonons and are, therefore, approximately elastic [5].

For an isotropic electron distribution, the occupation number $n^{\sigma}(\epsilon, t)$ of a spin-up $(\sigma=\uparrow)$ or spin-down $(\sigma=\downarrow)$

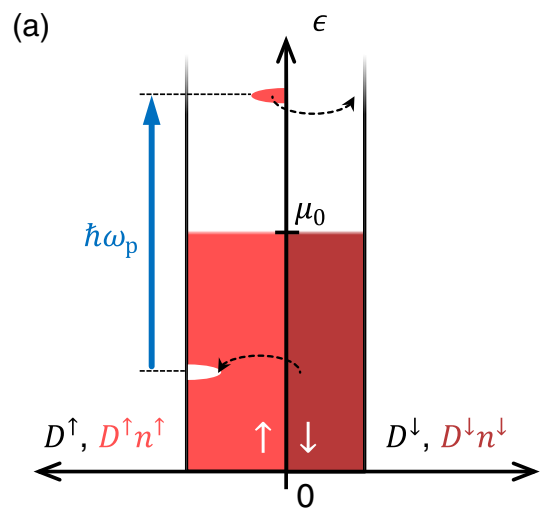

Bloch state depends only on its energy $\epsilon$ and on time $t$. As suggested by Fig. 4(a), the rate of change of the electron occupation $n^{\uparrow}(\epsilon, t)$ of a spin-up Bloch state with energy $\epsilon$ due to elastic spin-flip scattering is proportional to $n^{\uparrow} D^{\uparrow}$ and the number $\left(1-n^{\downarrow}\right) D^{\downarrow}$ of unoccupied spin-down states at the same energy $\epsilon$ minus an analogous term for the reverse process. Here, $D^{\sigma}(\epsilon)$ is the spin-dependent electronic density of states, and each transition is weighted with its squared matrix element $P_{\mathrm{sf}}(\epsilon)$. The rate of change in the magnetization $M \propto \int d \epsilon\left(D^{\uparrow} n^{\uparrow}-D^{\downarrow} n^{\downarrow}\right)$ is, therefore, given by

$$
\dot{M} \propto-2 \int d \epsilon\left(\Delta n^{\uparrow}-\Delta n^{\downarrow}\right) D^{\uparrow} D^{\downarrow} P_{\mathrm{sf}},
$$

where $\Delta n^{\sigma}(\epsilon, t)$ denotes the pump-induced change in the occupation number $n^{\sigma}$. In the linear fluence regime of our experiment (see Fig. S4 [35]), the weighting factor $\left(D^{\uparrow} D^{\downarrow} P_{\mathrm{sf}}\right)(\epsilon, t)$ can be evaluated for the unexcited sample and is, thus, time independent.

To explore Eq. (3), we consider two instructive scenarios right after excitation by the pump (photon energy $\hbar \omega_{p}$ ). First, we assume that $P_{\mathrm{sf}}, D^{\uparrow}$, and $D^{\downarrow}$ are all independent of energy [Fig. 4(a)]. In the spin-up subset, the pump generates holes below and electrons above the Fermi level $\mu_{0}$. Because $P_{\text {sf }} D^{\uparrow} D^{\downarrow}$ is constant with respect to $\epsilon$, a spin-flip transition of an excited spin-up electron into a spin-down state is as likely as that of an excited spin-up hole. An analogous argumentation for the spin-down channel shows that no net magnetization change occurs. Therefore, an

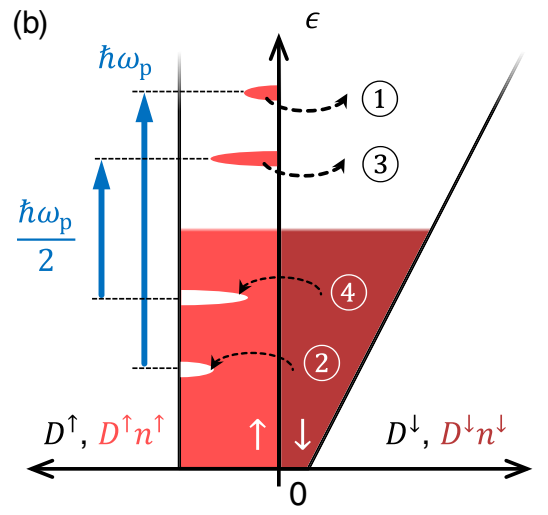

FIG. 4. Two model scenarios of optically induced magnetization quenching. (a) The electronic densities of states $D^{\uparrow}(\epsilon)$ and $D^{\downarrow}(\epsilon)$ (black lines) and transition-matrix elements are independent of electron energy $\epsilon$. The total number $n^{\sigma}(\epsilon) D^{\sigma}(\epsilon)$ of electrons with spin $\sigma=\uparrow$ or $\downarrow$ and energy $\epsilon$ is indicated by the red area. The pump pulse (photon energy $\hbar \omega_{p}$, blue arrow) generates electrons and holes above and below the Fermi level $\mu_{0}$, thereby modifying the number of spin-up electrons $D^{\uparrow} n^{\uparrow}$ at a given energy $\epsilon$. Because $D^{\uparrow}$ and $D^{\downarrow}$ do not depend on $\epsilon$, the net number of spin-flip scattering events is zero, leaving the magnetization unchanged. (b) The spin-down density of states $D^{\downarrow}$ increases linearly with $\epsilon$. Consequently, more scattering events for the spin-up electrons (1) than for the spin-up holes (2) are possible, resulting in magnetization quenching. When the photon energy is reduced to, for instance, $\hbar \omega_{p} / 2$, the difference in the number of final spin-down states for a spin-up electron (3) minus the analogous number for a spin-up hole (4) decreases by a factor of 2. This effect is, however, compensated by the number of pump-excited spin-up electrons, which has increased by a factor of 2. The resulting demagnetization rate is the same as for photon energy $\hbar \omega_{p}$, provided the deposited pump energy is the same. 
energy-dependent product $D^{\uparrow} D^{\downarrow} P_{\text {sf }}$ is essential to obtain a magnetization change.

Consequently, in the second scenario [Fig. 4(b)], we allow the spin-down density of states to depend on energy linearly, that is, $D^{\downarrow}(\epsilon)=D^{\downarrow}\left(\mu_{0}\right)+\gamma^{\downarrow} \cdot\left(\epsilon-\mu_{0}\right)$. From Fig. 4(b), we see that the excited spin-up electrons can scatter into a larger number of spin-down states than the excited spin-up holes. Therefore, the monotonic increase of $D^{\downarrow}(\epsilon)$ with energy $\epsilon$ enables magnetization quenching.

Note that the number of final spin-down states for spinup electrons minus the analogous number for spin-up holes scales with $D^{\downarrow}\left(\epsilon+\hbar \omega_{p}\right)-D^{\downarrow}(\epsilon)=\gamma^{\downarrow} \hbar \omega_{p}$. On the other hand, the number of pump-excited spin-up electrons is proportional to $W_{p}^{\uparrow} / \hbar \omega_{p}$, where $W_{p}^{\uparrow}$ is the pump-pulse energy that is absorbed by spin-up electrons. Therefore, the impact of the pump photon energy $\hbar \omega_{p}$ cancels, and the demagnetization rate is determined only by $W_{p}^{\uparrow}$. This reasoning is illustrated in Fig. 4(b) for the two pump energies $\hbar \omega_{p}$ and $\hbar \omega_{p} / 2$.

The preceding consideration can be extended to any pump-induced variation $\Delta n^{\sigma}$ of the electron distribution (see Appendix C and Ref. [7]). Assuming that no magnetization quenching has occurred yet $(\Delta M=0)$, we find that the quenching rate $\Delta \dot{M}$ is proportional to a weighted average of the energy the pump pulse has deposited in the spin-up and spin-down electrons, independent of the precise shape of the electron distribution [see Eqs. (C18) and $(\mathrm{C} 21)]$.

More generally, one can assign generalized temperatures $T_{0}+\Delta \tilde{T}^{\sigma}(t)$ to the instantaneous electron distributions $n_{0}+\Delta n^{\sigma}(\epsilon, t)$. They reduce to the familiar temperatures when the two distributions $n_{0}+\Delta n^{\sigma}$ are Fermi-Dirac functions [see Eq. (C8)]. Assuming that the generalized temperatures of spin-up and spin-down electrons equilibrate rapidly, the electrons can be described by one common excess temperature $\Delta \tilde{T}_{e}=\Delta \tilde{T}^{\uparrow}=\Delta \tilde{T}^{\downarrow}$, which is directly proportional to the instantaneous excess energy of all electrons [see Eq. (C21)]. In this case, the evolution of the magnetization can be shown [Eq. (C20)] to proceed according to

$$
\Delta M(t) \propto \int_{0}^{\infty} d \tau e^{-\Gamma_{\mathrm{es}} \tau} \Delta \tilde{T}_{e}(t-\tau)
$$

where $\Gamma_{\text {es }}$ is the inverse time constant of electron-spin equilibration. If, for example, $\Delta \tilde{T}_{e}$ changes steplike, $\Delta M$ will adapt to the new temperature with time constant $\Gamma_{\mathrm{es}}^{-1}$.

The dynamics of $\Delta \tilde{T}_{e}$ can be described by the familiar two-temperature model [48], even though $T_{0}+\Delta \tilde{T}_{e}$ is a generalized temperature [see Eq. (C25)]. We conclude that the dynamics of $\Delta \tilde{T}_{e}$ and, thus, $\Delta M$ are determined by the electronic excess energy, independent of the way it was deposited.

\section{Dynamics of UDM}

To extract the pure magnetization dynamics $\Delta \boldsymbol{M}(t)=$ $\Delta M(t) \boldsymbol{u}_{y}$ from the transient MOKE rotation and ellipticity signals in Fig. 3, we use Eq. (1) and the procedure described in Ref. [43] and Appendix B. The resulting $\Delta M(t) / M_{0}$ curves are displayed in Fig. 5. As expected from the MOKE signals (Fig. 3), $\Delta M(t)$ proceeds almost identically for optical and $\mathrm{THz}$ excitation. The slight differences in the observed dynamics may appear due to uncertainties in the $\Delta M(t)$ extraction and deconvolution.

To compare the experimentally determined $\Delta M(t)$ with our model result [Eq. (4)], we calculate the dynamics of the electronic excess energy assuming a $\delta(t)$-like pump pulse. As detailed in Appendix $\mathrm{C}$, we obtain $\Delta \tilde{T}_{e}(t) \propto \Theta(t)\left[(1-R) e^{-\Gamma_{\mathrm{ep}} t}+R\right]$, where $\Theta(t)$ is the Heaviside step function, $\Gamma_{\mathrm{ep}}^{-1}$ is the time constant of electron-phonon equilibration, and $R$ is the ratio of electronic and total heat capacity of the sample. With this input, Eq. (4) yields

$\Delta M(t) \propto \Theta(t)\left[\frac{\Gamma_{\mathrm{es}}-R \Gamma_{\mathrm{ep}}}{\Gamma_{\mathrm{es}}-\Gamma_{\mathrm{ep}}} e^{-\Gamma_{\mathrm{es}} t}-\frac{(1-R) \Gamma_{\mathrm{es}}}{\Gamma_{\mathrm{es}}-\Gamma_{\mathrm{ep}}} e^{-\Gamma_{\mathrm{ep}} t}-R\right]$.

To account for our non- $\delta(t)$-like pump pulse, Eq. (5) is convoluted with the pump-intensity profile $\boldsymbol{E}_{\text {fic }}^{2}(t)$ (Fig. 3) analogous to Eq. (2). We fit the resulting function to the measured $\Delta M(t)$ jointly for both $\mathrm{THz}$ and optical pump, where $\Gamma_{\mathrm{es}}, \Gamma_{\mathrm{ep}}, R$, a global scaling factor, and a rigid time

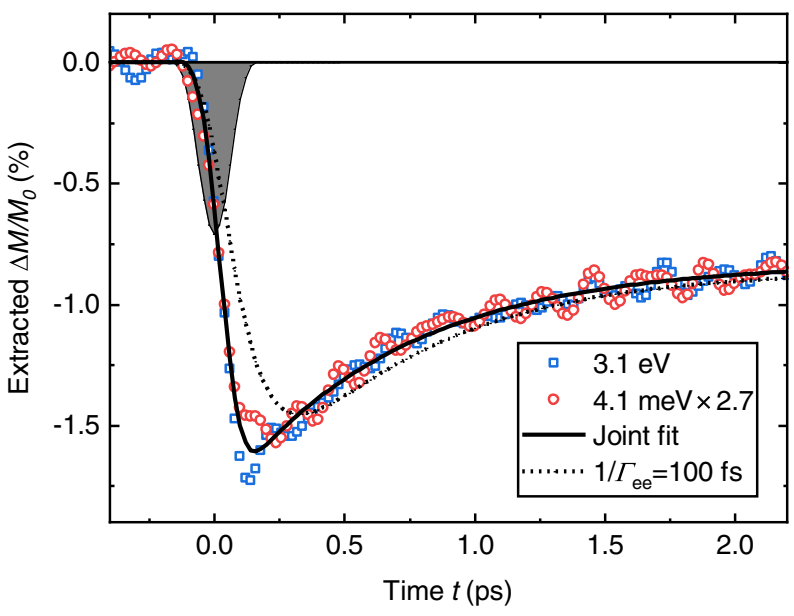

FIG. 5. Extracted magnetization dynamics $\Delta M(t) / M_{0}$ for optical (3.1 eV, blue squares) and $\mathrm{THz}(4.1 \mathrm{meV}$, red circles) excitation with a temporal profile indicated by the gray area. The data are inferred from the transients shown in Fig. 3. The black solid curve is a fit according to Eq. (5). The dotted curve is a result of a fit for $\mathrm{THz}$ excitation and subsequent convolution with a steplike exponential (decay time $100 \mathrm{fs}$ ) mimicking a situation where electron-electron thermalization after optical excitation is relevant (see Sec. IV). 
shift are free parameters. For the best fit (black solid curve in Fig. 5), we obtain $\Gamma_{\mathrm{es}}^{-1}=27 \pm 9$ fs, $\Gamma_{\text {ep }}^{-1}=670 \pm 50 \mathrm{fs}$, and $R=0.46 \pm 0.01$.

The value of $\Gamma_{\mathrm{es}}^{-1}$ is approximately compatible with the electron-spin equilibration time found previously [4]. We note, however, that the value of $\Gamma_{\mathrm{es}}^{-1}$ comes with significant uncertainty, because it heavily relies on the procedure we use to extract $\Delta M(t)$ and because the duration of our fictitious pump pulse is significantly longer. The extracted value of $\Gamma_{\mathrm{ep}}^{-1}$ is consistent with measurements of transient anisotropic reflectance $\Delta b(t)$ [see Eq. (1) and Fig. S7 [35]], whose decay is a monitor of electron-phonon equilibration [22], and with results from time-resolved photoelectron spectroscopy [6].

The value of $R$ obtained from the fit is much larger than the ratio of electronic and total heat capacity, which is about 0.06 for Fe [49]. This discrepancy is quite common for MOKE probing of UDM [4,5] and arises from the relatively large signal amplitude that remains after electrons and phonons have equilibrated ( $t>2 \mathrm{ps}$ in Figs. 3 and 5). We attribute this effect to the pump-induced variation $\Delta a_{y}$ of the magneto-optic coefficients [Eq. (1)]. As detailed in Appendix B, the extraction procedure [43] of $\Delta M=$ $\Delta M_{y}$ is designed to remove contributions of the increased electron temperature to $\Delta a_{y}$, which prevail directly after sample excitation [see Eq. (B9)]. However, the procedure does not cancel contributions due to the increased phonon temperature, which may lead to a significant vertical offset of the signal after excitation [see Appendix B and Eq. (B10)].

\section{DISCUSSION}

We observe almost identical magnetization dynamics of a Fe thin film following excitation by an optical and by a THz pump pulse (see Figs. 3 and 5). This behavior is remarkable, because the two pump pulses induce a very different transient state of the material. First, the THz pump pulse (photon energy of $4.1 \mathrm{meV}$ ) generates 3 orders of magnitude more electron-hole pairs than the optical pump (3.1 eV). Second, while excitation by the THz pulse merely results in a Fermi-Dirac electron distribution at elevated temperature, the optical pump induces a highly nonthermal electron distribution [Fig. 1(b)]. We are able to explain the identical dynamics following optical versus $\mathrm{THz}$ excitation by phase-space arguments for quasielastic spin-flip scattering [Fig. 4(b)].

\section{A. Impact of electron thermalization}

Note that identical dynamics following optical versus $\mathrm{THz}$ excitation would also be observed if the optically induced nonthermal electron distribution relaxed (thermalized) to a Fermi-Dirac-type distribution much faster than the time resolution (approximately $130 \mathrm{fs}$ ) of our experiment. This scenario appears, however, rather unlikely in view of known electron thermalization times $\Gamma_{\mathrm{ee}}^{-1}$ of transition metals such as $\mathrm{Ni}$ (approximately $100 \mathrm{fs}$ ) [50,51], Fe (approximately $100 \mathrm{fs}$ ) [52], Ru (approximately $140 \mathrm{fs}$ ) [21], Pt (approximately $100 \mathrm{fs}$ ) [22], Au (approximately $1 \mathrm{ps)} \mathrm{[53],} \mathrm{or} \mathrm{other} \mathrm{metals} \mathrm{like} \mathrm{Gd} \mathrm{(approximately}$ $100 \mathrm{fs})$ [20].

If electron thermalization was relevant for UDM, we would have to convolute the generalized temperature in Eq. (4) with a function that delays the rise of $\Delta \tilde{T}_{e}$ by $\Gamma_{\mathrm{ee}}^{-1}=100$ fs. To mimic this behavior, we first fit the THzexcitation data (for which electron thermalization is not relevant) in Fig. 5 with Eq. (5) and subsequently convolute the best fit by $H_{\mathrm{ee}}(t)=\Gamma_{\mathrm{ee}} \Theta(t) e^{-\Gamma_{\mathrm{ee}} t}$. The resulting curve (black dotted line) drops significantly more slowly than the measured one and, thus, cannot describe the opticalexcitation data. We conclude that electron thermalization is not relevant for UDM of $\mathrm{Fe}$, consistent with our theoretical model [Eq. (4)].

Interestingly, the difference between rotation and ellipticity signals shows that pump-induced changes $\Delta \boldsymbol{a}(t)$ in the magneto-optic coefficients contribute significantly to the measured MOKE signal, also after deconvolution (Fig. 3). This observation and the identical observed dynamics of the MOKE signals following optical and $\mathrm{THz}$ excitation (Fig. 3) suggest that $\Delta \boldsymbol{a}(t)$ also scales with the electronic excess energy, independent of the shape of the electron and, possibly, phonon distribution. An analogous statement can be made for the dynamics of the nonmagnetic signal contribution $\Delta b(t)$ [see Eq. (1) and Fig. S7 [35]].

We emphasize that thermal and nonthermal excitation of metals do not always induce identical spin dynamics. An important example is the ultrafast interfacial spin Seebeck effect in stacks of yttrium iron garnet (YIG) and Pt thin films. Excitation of the Pt layer leads to a spin current from YIG to Pt, whose magnitude is the largest when the excited electron distribution in Pt relaxes to a Fermi-Dirac distribution [22].

\section{B. Other materials}

To test whether our observations for $\mathrm{Fe}$ are general or rather specific to $\mathrm{Fe}$, analog pump-probe experiments should be conducted on other transition-metal ferromagnets. Here, $\mathrm{Ni}$ is a particularly interesting case, because it is a strong ferromagnet, in contrast to the weak ferromagnet Fe. However, the MOKE response of $\mathrm{Ni}$ is a factor of 5 smaller than that of $\mathrm{Fe}$ at our $800-\mathrm{nm}$ probe wavelength [54]. Thus, the resulting signal levels are at the edge of our high probe sensitivity, which is close to the shot noise of the probe pulses. Other detection schemes such as single-shot detection may overcome this limitation.

Nevertheless, two arguments suggest that our observations for Fe carry over to other metallic ferromagnets such as Co and Ni. First, recent experiments [7,8] reveal that $\mathrm{UDM}$ of transition-metal ferromagnets and $\mathrm{THz}$ spin transport (TST) out of the magnet proceed with identical dynamics. Therefore, insights gained into the dynamics of 
TST can be transferred to UDM and vice versa. In particular, works on TST out of Fe [12], $\mathrm{CoFeB}$ [13], and $\mathrm{Ni}_{80} \mathrm{Fe}_{20}$ [55] show that the transport dynamics are independent of the pump photon energy over the ranges $3.1-1.55,3.1-0.8$, and $3.1-1.55 \mathrm{eV}$, respectively. Although these photon energies are certainly not yet in the THz range (approximately $4 \mathrm{meV}$ ), the results provide some indication that TST and, thus, UDM are independent of the pump photon energy for Fe-, Co-, and Ni-dominated magnets. Second, our modeling predicts identical magnetization dynamics following both optical and $\mathrm{THz}$ excitation not only for Fe, but also other ferromagnets such as $\mathrm{Co}$ and Ni.

\section{Model limitations}

It is important to note that our model relies on the moment expansion of Eq. (C7): The energy dependence of the weight function $W(\epsilon)=-2\left(P_{\mathrm{sf}} D^{\uparrow} D^{\downarrow}\right)(\epsilon)$ [see Eq. (3)] is assumed to depend linearly on the electron energy $\epsilon$ around the Fermi level $\mu_{0}$ of the unexcited sample. In other words, we approximate $W(\epsilon)$ by $W\left(\mu_{0}\right)+W^{\prime}\left(\mu_{0}\right)\left(\epsilon-\mu_{0}\right)$, which needs to hold over the width of the transient changes $\Delta n^{\uparrow}$ and $\Delta n^{\downarrow}$ in the distribution functions. Directly after optical excitation, this width is of the order of twice the pump photon energy, that is, $6.2 \mathrm{eV}$. Given calculated electronic densities of states of $\mathrm{Fe}, \mathrm{Co}$, and $\mathrm{Ni}$ [56], it is questionable whether $W(\epsilon)$ varies linearly over such a large energy range. Note, however, that the width of the transient electron distribution reduces by a factor of the order of 2 in the course of each electron-electron scattering cascade. From the $\mathrm{THz}$ conductivity of our sample, the electron scattering time is roughly estimated to be $10 \mathrm{fs}$ or less. Thus, only $30 \mathrm{fs}$ after optical excitation, the width of the transient electron distribution is expected to be significantly less than $1 \mathrm{eV}$, thereby making linearization of $W(\epsilon)$ and, thus, the moment expansion of Eq. (C7) much more credible.

From the experimental side, we note that if the moment expansion was strongly violated due to discontinuities of $W(\epsilon)$ around the Fermi energy, UDM and TST should proceed with different temporal dynamics [7]. However, for metallic magnets such as $\mathrm{Co}_{70} \mathrm{Fe}_{30}, \mathrm{Co}_{40} \mathrm{Fe}_{40} \mathrm{~B}_{20}$, and $\mathrm{Ni}_{80} \mathrm{Fe}_{20}$, TST and UDM are observed to follow identical temporal evolution [7]. We, thus, conclude that the moment expansion of Eq. (C7) is fulfilled for $\mathrm{Co}_{-}, \mathrm{Fe}-$, and Ni-dominated ferromagnetic alloys.

\section{Conclusions}

We have shown that both optical and $\mathrm{THz}$ excitation of a ferromagnetic $\mathrm{Fe}$ thin film result in identical magnetization dynamics, even though the two pulses induce very different changes in the electron distribution directly after absorption. Phase-space considerations of quasielastic spin-flip scattering can consistently explain our observations. They show that the UDM dynamics are determined by the electronic excess energy, independent of the way it is deposited.

Our results are also relevant from an applied viewpoint. First, the speed of UDM is not limited by carrier multiplication [22]. The onset of its rate is determined only by the pump-pulse duration. Second, because ultrafast spin transport is driven by the same force as UDM [6-9], it should exhibit the same dynamics, independent of the photon energy of the pump pulse. Such behavior was indeed reported recently for photon energies between 0.8 and $3.1 \mathrm{eV}[12,13,55]$. Our insights are, in particular, interesting for two practical applications involving UDM and, thus, ultrafast spin transport: all-optical helicitydependent magnetization switching [2] and spintronic generation of broadband $\mathrm{THz}$ pulses [11-13].

\section{ACKNOWLEDGMENTS}

We acknowledge funding by the German Research Foundation through the collaborative research center SFB TRR 227 "Ultrafast spin dynamics" (Projects A01, $\mathrm{A} 05, \mathrm{~B} 02$, and B03) and the European Union through the ERC H2020 CoG project TERAMAG/Grant No. 681917.

\section{APPENDIX A: EXPERIMENTAL DETAILS}

\section{Setup details}

For generation of the pump pulses, we use an amplified Ti:sapphire system. Optical pump pulses (spot diameter $200 \mu \mathrm{m}$, photon energy $3.1 \mathrm{eV}$, repetition rate $1 \mathrm{kHz}$, and $p$-polarized) are generated by frequency doubling of the laser output in a BBO crystal. The $\mathrm{THz}$ pulses $(900 \mu \mathrm{m}$, $4.1 \mathrm{meV}, 1 \mathrm{kHz}$, and $p$-polarized) are generated by tiltedwave-front-type optical rectification in a $\mathrm{LiNbO}_{3}$ crystal and focused on the sample using three parabolic mirrors (see Fig. S1a [35]). For the probe pulses, we use the output of the amplified system seed oscillator $(100 \mu \mathrm{m}, 1.6 \mathrm{eV}$, $80 \mathrm{MHz}$, and polarized at $3^{\circ}$ out of the $s$ axis).

The paths of probe and optical pump are combined by means of a dichroic mirror. The probe is reflected from the magnetized sample [Fig. 1(a)] and directed into the balanced detection. An optional quarter wave plate serves to switch between measurements of probe rotation and ellipticity. The polarity of the external magnetic field (magnitude $40 \mathrm{mT}$ ) is alternated at a rate of $500 \mathrm{~Hz}$. We use two $\mathrm{THz}$ polarizers to alternate the polarity of the $\mathrm{THz}$ pulse. A set of filters serves to block the fundamental after the $\mathrm{BBO}$ crystal and to block the optical pump after reflection from the sample.

\section{Pump-pulse characterization}

For our deconvolution procedure (see Appendix B), we need to know the intensity profiles $\boldsymbol{E}_{p}^{2}(t)$ of the pump pulses versus time. For the $\mathrm{THz}$ pump, we calculate $\boldsymbol{E}_{p}^{2}(t) \propto \boldsymbol{B}_{p}^{2}(t)$ from the transient $\mathrm{THz}$ magnetic field 
$\boldsymbol{B}_{p}(t)$, which is obtained from the Zeeman-torque contribution [see Fig. 2(b)]. For comparison, we also measure the $\mathrm{THz}$ electric field $\boldsymbol{E}_{p}(t)$ by electro-optic sampling in quartz [see Fig. S3(b) [35]] [36]. For the optical pump, we measure $\boldsymbol{E}_{p}^{2}(t)$ through the optical Kerr effect in a 300- $\mu$ m-thick diamond window [see Figs. 2(c) and S3(c) [35]].

\section{Sample details}

The Fe thin film is grown by electron-beam evaporation at a rate of $0.2 \AA \mathrm{s}^{-1}$ and a base pressure in the $10^{-9} \mathrm{mbar}$ range. The film has in-plane magnetic anisotropy. Hysteresis loops of $M_{y}$ as measured by the longitudinal MOKE are shown in Fig. S1(b) [35].

The THz conductance of the Fe thin film is determined by $\mathrm{THz}$ transmission spectroscopy $[57,58]$, in which we measure $\mathrm{THz}$ fields $E_{\mathrm{Fe} \| \text { sub }}$ and $E_{\text {sub }}$ through the sample $\mathrm{Fe} \|$ sub (Fe film on substrate) and the substrate (sub) without $\mathrm{Fe}$ film, respectively. In the frequency domain, the ratio $T(\omega)$ of the two fields obeys

$T(\omega)=\frac{\tilde{E}_{\mathrm{Fe} \| \text { sub }}(\omega)}{\tilde{E}_{\mathrm{sub}}(\omega)}=\frac{n_{a}(\omega)+n_{\mathrm{sub}}(\omega)}{n_{a}(\omega)+n_{\mathrm{sub}}(\omega)+Z_{0} G(\omega)}$,

where $n_{a}(\omega) \approx 1$ and $n_{\text {sub }}(\omega) \approx 2.1$ are the known refractive indices of air and the glass substrate [59], respectively [see Fig. 2(a)], and $Z_{0} \approx 377 \Omega$ is the free-space impedance. The conductance $G(\omega)=\sigma(\omega) d$ of the nominally homogeneous $\mathrm{Fe}$ film is given by the product of its conductivity $\sigma(\omega)$ and thickness $d=4 \mathrm{~nm}$. We, thus, obtain

$$
G(\omega)=\frac{n_{a}(\omega)+n_{\mathrm{sub}}(\omega)}{Z_{0}}\left[\frac{1}{T(\omega)}-1\right],
$$

from which the conductivity $\sigma(\omega)=G(\omega) / d$ is obtained. Results of the $\mathrm{THz}$ transmission measurements are shown in Fig. S5 [35]. By fitting the conductivity with a Drude model [see Fig. S5(b) [35]], we obtain an electron velocity relaxation time of approximately $8 \mathrm{fs}$.

\section{Absorbed fluences}

To determine the $\mathrm{THz}$ absorptance $A_{\mathrm{THz}}(\omega)$ of the $\mathrm{Fe}$ film, we take advantage of the $\mathrm{THz}$ transmission measurements. The absorbed power per volume is given by $d P_{\mathrm{abs}} / d V=\left|\tilde{E}_{\mathrm{Fe}}(\omega)\right|^{2} \operatorname{Re}\{\sigma(\omega)\} / 2$. The approximately homogeneous $\mathrm{THz}$ pump field $\tilde{E}_{\mathrm{Fe}}(\omega)$ within and directly behind the Fe film is related to the incident field $\tilde{E}_{\text {inc }}(\omega)$ and the measured $T(\omega)$ [Eq. (A1)] through $\tilde{E}_{\mathrm{Fe}}(\omega)=$ $2 n_{a}(\omega) T(\omega) \tilde{E}_{\text {inc }}(\omega) /\left[n_{a}(\omega)+n_{\text {sub }}(\omega)\right]$, whereas $\sigma(\omega)=$ $G(\omega) / d$ is obtained from Eq. (A2). Integration of $d P_{\text {abs }} / d V$ from $z=0$ to $d$ yields the absorbed power per sample area:

$$
\begin{aligned}
\frac{d P_{\mathrm{abs}}}{d A}= & \frac{\left|\tilde{E}_{\mathrm{inc}}(\omega)\right|^{2}}{2 Z_{0}}\left|\frac{2 n_{a}(\omega) T(\omega)}{n_{a}(\omega)+n_{\mathrm{sub}}(\omega)}\right|^{2} \\
& \times \operatorname{Re}\left\{\left[\frac{1}{T(\omega)}-1\right]\left[n_{a}(\omega)+n_{\mathrm{sub}}(\omega)\right]\right\} .
\end{aligned}
$$

By assuming a real-valued $n_{a}(\omega)$, the power flux $d P_{\text {inc }} / d A$ of the incident wave can be written as $n_{a}(\omega)\left|\tilde{E}_{\text {inc }}(\omega)\right|^{2} /$ $2 Z_{0}$, and, with Eq. (A3), its absorbed fraction becomes

$$
\begin{aligned}
A_{\mathrm{THz}}(\omega)= & \frac{d P_{\mathrm{abs}} / d A}{d P_{\mathrm{inc}} / d A}=n_{a}(\omega)\left|\frac{2 T(\omega)}{n_{a}(\omega)+n_{\mathrm{sub}}(\omega)}\right|^{2} \\
& \times \operatorname{Re}\left\{\left[\frac{1}{T(\omega)}-1\right]\left[n_{a}(\omega)+n_{\mathrm{sub}}(\omega)\right]\right\} .
\end{aligned}
$$

We obtain $A_{\mathrm{THz}} \approx 0.3$ for normal incidence [Fig. S5(a) [35]].

Because the pump-induced MOKE signal $\Delta S^{\boldsymbol{M}_{0}, \boldsymbol{E}_{p}^{2}}$ [see Eq. (B1)] depends linearly on the pump fluence (see Fig. S4 [35]), the ratio of the pump-probe signals long after excitation should be equal to the ratio of absorbed fluences $F_{\text {opt }} A_{\text {opt }} / F_{\mathrm{THz}} A_{\mathrm{THz}}$, where $F_{\text {opt }}$ and $F_{\mathrm{THz}}$ is the incident fluence and $A_{\mathrm{opt}}$ and $A_{\mathrm{THz}}$ is the absorptance of the Fe film. From the incident powers and approximate pumpspot sizes, we calculate incident fluences of $F_{\text {opt }}=$ $0.15 \mathrm{~mJ} \mathrm{~cm}^{-2}$ and $F_{\mathrm{THz}}=0.06 \mathrm{~mJ} \mathrm{~cm}^{-2}$. We estimate the absorptances for the case of normal incidence and obtain $A_{\text {opt }} \approx 0.4$ [11] and $A_{\mathrm{THz}} \approx 0.3$ (see above). The result is $F_{\text {opt }} A_{\text {opt }} / F_{\mathrm{THz}} A_{\mathrm{THz}} \approx 3.6$, which is in reasonable agreement with the experimental value of 2.7 (see Fig. 3).

\section{APPENDIX B: PUMP-PROBE DATA ANALYSIS}

\section{Processing of raw signals}

The measured pump-induced signals are probe rotation or ellipticity changes [see Fig. S1(a) [35]] with respect to the equilibrium value, when no pump is applied: $\Delta S(t)=S(t)-S_{0}$. The signals are recorded for two opposite magnetizations $\pm \boldsymbol{M}_{0}$ of the Fe film and two opposite pump fields $\pm \boldsymbol{E}_{p}(t)$ in the case of $\mathrm{THz}$ pumping [Figs. S2(a) and S2(b) [35]]. To separate various contributions by their parity with respect to $\boldsymbol{M}_{0}$ and $\boldsymbol{E}_{p}$, we take corresponding sums (even parity) and differences (odd parity) of these signals [33]. For example, to separate the contribution $\Delta S^{\boldsymbol{M}_{0}, \boldsymbol{E}_{p}}$ linear in $\boldsymbol{M}_{0}$ and linear in $\boldsymbol{E}_{p}$ (which is dominated by Zeeman torque) and contribution $\Delta S^{\boldsymbol{M}_{0}, \boldsymbol{E}_{p}^{2}}$ linear in $\boldsymbol{M}_{0}$ yet quadratic in $\boldsymbol{E}_{p}$ (which corresponds to UDM), we use

$$
\left.\begin{array}{rl}
4 \Delta S^{\boldsymbol{M}_{0}, \boldsymbol{E}_{p}}(t) \\
4 \Delta S^{\boldsymbol{M}_{0}, \boldsymbol{E}_{p}^{2}}(t)
\end{array}\right\}=\left[\begin{array}{l}
{\left[\Delta\left(t,+\boldsymbol{M}_{0},+\boldsymbol{E}_{p}\right)-\Delta S\left(t,-\boldsymbol{M}_{0},+\boldsymbol{E}_{p}\right)\right]} \\
\\
\mp\left[\Delta S\left(t,+\boldsymbol{M}_{0},-\boldsymbol{E}_{p}\right)-\Delta S\left(t,-\boldsymbol{M}_{0},-\boldsymbol{E}_{p}\right)\right]
\end{array}\right.
$$


and similarly for all other signal components [Figs. S2(c) and S2(d) [35]]. The signals corresponding to UDM can be normalized by the height of the MOKE hysteresis loop given by $S_{0}\left(\boldsymbol{M}_{0}\right)-S_{0}\left(-\boldsymbol{M}_{0}\right)=2 a_{0 y} M_{0}$. Based on Eq. (1), such normalization yields relative variations of magnetization and magneto-optic coefficient:

$$
\begin{aligned}
\frac{2 \Delta S^{\boldsymbol{M}_{0}, \boldsymbol{E}_{p}^{2}}(t)}{S_{0}\left(+\boldsymbol{M}_{0}\right)-S_{0}\left(-\boldsymbol{M}_{0}\right)} & =\frac{\boldsymbol{a}_{0}}{a_{0 y}} \cdot \frac{\Delta \boldsymbol{M}(t)}{M_{0}}+\frac{\Delta a_{y}(t)}{a_{0 y}} \\
& =\frac{\Delta M_{y}(t)}{M_{0}}+\frac{\Delta a_{y}(t)}{a_{0 y}} .
\end{aligned}
$$

Similar to the odd-in- $\boldsymbol{M}_{0}$ responses, we perform the analysis for the nonmagnetic responses (even in $\boldsymbol{M}_{0}$ and $\boldsymbol{E}_{p}$ ) and directly compare them for optical and $\mathrm{THz}$ pump.

\section{Deconvolution procedure}

According to Eq. (2), the measured pump-probe signal $\Delta S(t)$ is a convolution of the intensity profile of the driving pulse $\boldsymbol{E}_{p}^{2}(t)$ and the impulse-response function $H(t)$, which can be interpreted as the system response to a $\delta$-like pump pulse. By applying Fourier transformation to Eq. (2), we obtain

$$
\Delta \widetilde{S}(\omega)=\widetilde{H}(\omega) \widetilde{\boldsymbol{E}_{p}^{2}}(\omega),
$$

where the tilde denotes the Fourier transformation. Practically, it is not possible to solve Eq. (B3) for $\tilde{H}(\omega)$ at all frequencies $\omega / 2 \pi$, because the width of the spectrum of $\boldsymbol{E}_{p}^{2}$ is finite. Therefore, we rather consider the system response to a fictitious pump pulse with intensity profile $\boldsymbol{E}_{\text {fic }}^{2}(t)$ and obtain

$$
\Delta \widetilde{S}_{\mathrm{dec}}(\omega)=\widetilde{H}(\omega) \widetilde{\boldsymbol{E}_{\mathrm{fic}}^{2}}(\omega)=\Delta \widetilde{S}(\omega) \frac{\widetilde{\boldsymbol{E}_{\mathrm{fic}}^{2}}(\omega)}{\widetilde{\boldsymbol{E}_{\mathrm{p}}^{2}}(\omega)} .
$$

For the spectrum of $\boldsymbol{E}_{\text {fic }}^{2}$, we set $\widetilde{\boldsymbol{E}_{\text {fic }}^{2}}(\omega)=F\left(\omega / \omega_{c}\right)$, where $F\left(\omega / \omega_{c}\right)$ is zero for frequencies $|\omega|$ above the cutoff frequency $\omega_{c}$. Simultaneously, in the time domain, $\boldsymbol{E}_{\text {fic }}^{2}(t)$ should consist of only one peak at $t=0$ and decay rapidly to zero with negligible side lobes. These requirements are excellently fulfilled by the Norton-Beer apodizing function of Eq. (3) in Ref. [60] for the case "FWHM = 2.0." For $|x| \leq 1$, it is given by

$$
F(x)=\sum_{j=0}^{8} C_{j}\left(1-x^{2}\right)^{j}
$$

while $F(x)=0$ otherwise. The nonvanishing coefficients in Eq. (B5) are $C_{0}=0.002267, C_{2}=0.140412$, $C_{4}=0.487172, \quad C_{6}=0.256200$, and $C_{8}=0.113948$
[60]. The spectrum $\widetilde{\boldsymbol{E}_{\text {fic }}^{2}}(\omega)$ and time-domain trace $\boldsymbol{E}_{\text {fic }}^{2}(t)$ are, respectively, shown in Figs. S6(b) and S6(a) [35] for a cutoff frequency $\omega_{c} / 2 \pi$ of $8 \mathrm{THz}$.

Inverse Fourier transformation of Eq. (B4) finally yields $\Delta S_{\mathrm{dec}}(t)=\left(H * \boldsymbol{E}_{\mathrm{fic}}^{2}\right)(t)$, the system response to the fictitious pump pulse. As our choice of $\boldsymbol{E}_{\mathrm{fic}}^{2}$ is essentially nonnegative and well localized, the signal $\Delta S_{\mathrm{dec}}(t)$ can be understood as the response $H$ to a $\delta$-like pump pulse, which is subsequently smeared over the width of $\boldsymbol{E}_{\text {fic }}^{2}(t)$.

To obtain the finest time resolution of $\Delta S_{\mathrm{dec}}$, the cutoff frequency $\omega_{c} / 2 \pi$ should be as large as possible. However, when $\omega_{c}$ is increased, it is finally located in the noise floor of the spectrum $\boldsymbol{E}_{p}^{2}$ of the pump-intensity trace. The deconvolution [Eq. (B4)] tends to amplify this noise, resulting in oscillations in the time domain. This effect can be observed in Fig. S6(c) [35]: With increasing $\omega_{c}$, the rising edge of $\Delta S_{\mathrm{dec}}$ becomes steeper, but oscillatory features appear.

\section{Extracting magnetization dynamics}

For $\Delta M_{x}=\Delta M_{z}=0$, the relative pump-induced change in the MOKE signal can, according to Eq. (B2), be rewritten as

$$
\delta S(t)=\delta M(t)+\delta a(t),
$$

where $\delta S(t)$ equals the left-hand side of Eq. (B2), $\delta M(t)=\Delta M_{y} / M_{0}$, and $\delta a(t)=\Delta a_{y} / a_{y 0}$. As seen in Fig. 2, the contribution $\delta a(t)$ leads to different dynamics of rotation and ellipticity signals because, in general, $\delta a^{\text {rot }}(t) \neq \delta a^{\text {ell }}(t)$. To separate the components $\delta M(t)$ and $\delta a(t)$ of our signal, we follow Ref. [43] and assume that

$$
\delta a^{\mathrm{rot}}(t)=g_{1} \delta a^{\mathrm{ell}}(t),
$$

where $g_{1}$ is a proportionality constant. We have, thus, three equations for three unknowns, which yield

$$
\delta M(t)=\frac{\delta S^{\mathrm{rot}}(t)-g_{1} \delta S^{\mathrm{ell}}(t)}{1-g_{1}} .
$$

As in Ref. [43], we choose the value of the unknown constant $g$ such that the resulting signal $\delta M(t)$ does not exhibit discontinuities in the vicinity of $t=0$, that is, at the arrival time of the pump. Interestingly, the optimal value of $g_{1}$ is approximately -1 for both the optical and the $\mathrm{THz}$ pump, thereby resulting in simple summation of the two transients [see Eq. (B8)]. The resulting magnetization transients are shown in Fig. 5. The impact of various values of $g_{1}$ is shown in Fig. S8 [35].

In an attempt to justify and extend Eq. (B7), we note that the pump-induced changes $\delta a(t)$ in the magneto-optic coupling constants for small excitation densities are given by a linear functional of the changes in the occupation 
numbers of spin-up $(\sigma=\uparrow)$ and spin-down $(\sigma=\downarrow)$ electrons and in the phonon occupation numbers. Thus, following the moment expansion of Eq. (C7) in Appendix $\mathrm{C}$ below, one can characterize the electronic contribution to $\delta a$ by a linear combination of changes in the generalized electron temperatures $\Delta \tilde{T}^{\sigma}$ and chemical potentials $\Delta P^{\sigma}$. By definition, $\delta a$ does not depend on $\Delta M$ to linear order. Because of Eq. (C14), the $\Delta P^{\sigma}$ do not contribute to $\delta a$ to linear order, either. We finally assume that the temperatures quickly equilibrate $\left(\Delta \tilde{T}^{\uparrow}=\Delta \tilde{T}^{\downarrow}=\right.$ $\Delta \tilde{T}_{e}$ ) and that the phononic contribution to $\delta a$ is sufficiently characterized by the change $\Delta \tilde{T}_{p}$ in the generalized phonon temperature. This reasoning yields

$$
\delta a(t)=A_{e} \Delta \tilde{T}_{e}(t)+A_{p} \Delta \tilde{T}_{p}(t),
$$

where the constant coefficients $A_{e}$ and $A_{p}$ are, in general, different for MOKE rotation and ellipticity. Directly after excitation by a $\delta$-like pump pulse, $\Delta \tilde{T}_{e}$ is much larger than $\Delta \tilde{T}_{p}$, and Eq. (B9) approximately leads to $\delta a \propto \Delta \tilde{T}_{e}$ for both rotation and ellipticity, resulting in Eq. (B7). At longer times, however, the decrease of $\Delta \tilde{T}_{e}$ and increase of $\Delta \tilde{T}_{p}$ may cause a sizable contribution of the phonons to $\delta a$ and, thus, violate Eq. (B7).

To extend Eq. (B7) to longer times, we note that, for constant electron and phonon heat capacities, energy conservation implies $\Delta \tilde{T}_{p} \propto \Delta \tilde{T}_{e}$ for $t>0$. This relationship results in $\Delta \tilde{T}_{p}(t)=B_{1} \Delta \tilde{T}_{e}(t)+B_{2} \Theta(t)$, where $B_{1}$ and $B_{2}$ are constant coefficients and $\Theta(t)$ is the Heaviside step function. By substituting $\Delta \tilde{T}_{p}(t)$ in Eq. (B9) and accounting for the shape of the pump pulse analogous to Eq. (2), we obtain

$$
\delta a^{\mathrm{rot}}(t)=g_{1} \delta a^{\mathrm{ell}}(t)+g_{2} \cdot\left(\boldsymbol{\Theta} * \boldsymbol{E}_{p}^{2}\right)(t) .
$$

Finally, by substituting Eq. (B10) into the two Eqs. (B6) yields

$\delta M(t)=\frac{\delta S^{\mathrm{rot}}(t)-g_{1} \delta S^{\mathrm{ell}}(t)}{1-g_{1}}-\frac{g_{2}}{1-g_{1}}\left(\Theta * \boldsymbol{E}_{p}^{2}\right)(t)$.

In practical terms, Eq. (B11) implies that one just needs to add the function $\left(\Theta * \boldsymbol{E}_{p}^{2}\right)(t)$ with suitable overall amplitude to the previously used Eq. (B8) [43], thereby predominantly changing the signal strength at longer delays by an offset. For the fit using Eq. (5), this modification results in a different value of the fit parameter $R$.

\section{APPENDIX C: MODEL OF MAGNETIZATION DYNAMICS}

\section{Model details}

We adopt the model in Ref. [47], which accounts for ferromagnetism by a Stoner-type approach and treats the dynamics by Boltzmann-type rate equations. All spinconserving electron scattering events are considered, while for spin flips, we restrict to quasielastic single-electron scattering [5].

We assume that the state of the electronic system is fully characterized by the occupation numbers $n_{k}^{\sigma}$ of a Bloch state $(k, \sigma)$, where $\sigma=\uparrow, \downarrow$ refers to the electron spin and $k$ summarizes the band index and wave vector. We also assume that the $k$ dependence is fully given by the energy $\epsilon_{k}^{\sigma}$ of the Bloch state, that is,

$$
n_{k}^{\sigma}(t)=n^{\sigma}\left(\epsilon_{k}^{\sigma}, t\right) .
$$

In the framework of the Stoner model, the Bloch energy depends on the pump-induced change $\Delta m$ in the magnetic moment according to

$$
\epsilon_{k}^{\sigma}(t)=\epsilon_{k 0}^{\sigma}+I^{\sigma} \Delta m(t) .
$$

Here, $\epsilon_{k 0}^{\sigma}$ is the Bloch energy before arrival of the pump pulse, and $I^{\sigma}=I^{\uparrow, \downarrow}= \pm I / 2$ quantifies the strength of the effective electron-electron Coulomb interaction.

Note that we are interested only in effects up to first order in the pump-induced changes

$$
\Delta n^{\sigma}=n^{\sigma}-n_{0}
$$

in the distribution functions. Before arrival of the pump pulse, the $n^{\sigma}(\epsilon, t)$ are given by one and the same FermiDirac function $n_{0}(\epsilon)$ at temperature $T_{0}$. The reason is that, in our experiments, all demagnetization signals are found to scale linearly with the deposited pump-pulse energy.

The magnetic moment of the sample in Bohr magnetons is given by

$$
m=\int d \epsilon\left(D^{\uparrow} n^{\uparrow}-D^{\downarrow} n^{\downarrow}\right) .
$$

As derived in the main text, the rate of change in $m$ is given by Eq. (3), which is rewritten as

$$
\Delta \dot{m}=-2 \int d \epsilon\left(\Delta n^{\uparrow}-\Delta n^{\downarrow}\right) g_{\mathrm{sf}} .
$$

Here, $g_{\mathrm{sf}}(\epsilon)=\left(P_{\mathrm{sf}} D^{\uparrow} D^{\downarrow}\right)(\epsilon)$ scales with the probability that a spin-up electron with energy $\epsilon$ makes a transition into spin-down states of the same energy. Because the density $D^{\uparrow}$ and $D^{\downarrow}$ of, respectively, spin-up and spin-down electronic states and the squared matrix $P_{\text {sf }}$ element can all depend on the Bloch-electron energy $\epsilon$, the product $g_{\mathrm{sf}}$ does also.

\section{Moment expansion}

To analyze Eq. (C5), we assume that the weight factor $W=-2 g_{\text {sf }}$ can be approximated by 


$$
W(\epsilon)=W\left(\mu_{0}\right)+W^{\prime}\left(\mu_{0}\right)\left(\epsilon-\mu_{0}\right)
$$

for all energies $\epsilon$ around the unperturbed chemical potential $\mu_{0}$ at which the changes $\Delta n^{\uparrow}$ and $\Delta n^{\downarrow}$ in the electron distribution are notably nonzero. The integral over $W \Delta n^{\sigma}$ ( $\sigma=\uparrow, \downarrow)$ then yields

$$
\int d \epsilon W \Delta n^{\sigma}=W\left(\mu_{0}\right) \Delta P^{\sigma}+W^{\prime}\left(\mu_{0}\right) \Delta A^{\sigma},
$$

which is just a linear combination of the zeroth and first moments of the distribution changes $\Delta n^{\sigma}$. The moments are defined as

$$
\begin{aligned}
& \Delta P^{\sigma}=\int d \epsilon \Delta n^{\sigma} \quad \text { and } \\
& \Delta A^{\sigma}=\frac{\pi^{2} k_{B}^{2}}{3} T_{0} \Delta \tilde{T}^{\sigma}=\int d \epsilon\left(\epsilon-\mu_{0}\right) \Delta n^{\sigma} .
\end{aligned}
$$

In the case when $n^{\sigma}=n_{0}+\Delta n^{\sigma}$ is a Fermi-Dirac distribution with chemical potential $\mu^{\sigma}$ and temperature $T^{\sigma}, \Delta P^{\sigma}$ and $\Delta A^{\sigma}$ reduce to

$$
\Delta P^{\sigma}=\mu^{\sigma}-\mu_{0} \quad \text { and } \quad \Delta \tilde{T}^{\sigma}=T^{\sigma}-T_{0},
$$

respectively, to linear order in the $\Delta n^{\sigma}$. One can, thus, interpret $\Delta P^{\sigma}$ and $\Delta \tilde{T}^{\sigma} \propto \Delta A^{\sigma}$ as changes in, respectively, a generalized chemical potential and a generalized temperature. We emphasize, however, that the definition of the moments $\Delta P^{\sigma}$ and $\Delta A^{\sigma}$ [Eq. (C8)] also applies to nonthermal electron distributions $n_{0}+\Delta n^{\sigma}$. We use both $\Delta A^{\sigma}$ and $\Delta \tilde{T}^{\sigma}$ for convenience.

By applying the moment expansion of Eq. (C7) to Eq. (C5), we obtain

$$
\Delta \dot{m}=-2 g_{\mathrm{sf}}\left(\mu_{0}\right) \Delta P_{s}-2 g_{\mathrm{sf}}^{\prime}\left(\mu_{0}\right)\left(\Delta A^{\uparrow}-\Delta A^{\downarrow}\right) .
$$

The first term on the right-hand side describes magnetization relaxation driven by the spin voltage

$$
\Delta P_{s}=\Delta P^{\uparrow}-\Delta P^{\downarrow}
$$

The term proportional to $\Delta A^{\uparrow}-\Delta A^{\downarrow}$ is a term analogous to the Seebeck effect, which contributes as long as the generalized temperatures of spin-up and spin-down electrons are different.

\section{Magnetization dynamics}

To eliminate $\Delta P_{s}$ in Eq. (C10), we take advantage of particle conservation. The change in the sample magnetic moment [Eq. (C4)] is given by $\Delta m=\Delta\left(N^{\uparrow}-N^{\downarrow}\right)$, where $N^{\sigma}=\int d \epsilon D^{\sigma} n^{\sigma}$ is the number of electrons with spin $\sigma=\uparrow, \downarrow$. To linear order, the pump-induced change is

$$
\Delta N^{\sigma}=\int d \epsilon\left(\Delta D^{\sigma} n_{0}+D_{0}^{\sigma} \Delta n^{\sigma}\right)
$$

The pump-induced change $\Delta D^{\sigma}$ in the density of states arises from the magnetic-moment change through Eq. (C2). We express $\Delta D^{\sigma}(\epsilon)$ to first order in the $\Delta n^{\sigma}$ by $\Delta D^{\sigma}(\epsilon)=D_{0}^{\sigma \prime}(\epsilon) I^{\sigma} \Delta m$. By expanding $D_{0}^{\sigma}(\epsilon)=D_{0}^{\sigma}\left(\mu_{0}\right)+$ $D_{0}^{\sigma \prime}\left(\mu_{0}\right)\left(\epsilon-\mu_{0}\right)$ [see Eq. (C6)], we obtain $\int d \epsilon \Delta D^{\sigma} n_{0}=$ $D_{0}^{\sigma}\left(\mu_{0}\right) I^{\sigma} \Delta m$ for the first term of Eq. (C12). By applying the moment expansion [Eq. (C7)] to the second term, we find

$\Delta N^{\sigma}=D_{0}^{\sigma}\left(\mu_{0}\right) I^{\sigma} \Delta m+D_{0}^{\sigma}\left(\mu_{0}\right) \Delta P^{\sigma}+D_{0}^{\sigma \prime}\left(\mu_{0}\right) \Delta A^{\sigma}$.

By taking particle conservation into account, $\Delta\left(N^{\uparrow}+N^{\downarrow}\right)=0$, and making use of $\Delta m=\Delta\left(N^{\uparrow}-N^{\downarrow}\right)$ and $I^{\sigma}= \pm I / 2$, Eq. (C13) becomes

$\pm\left[1-I D_{0}^{\sigma}\left(\mu_{0}\right)\right] \Delta m=2 D_{0}^{\sigma}\left(\mu_{0}\right) \Delta P^{\sigma}+2 D_{0}^{\sigma \prime}\left(\mu_{0}\right) \Delta A^{\sigma}$

for $\sigma=\uparrow, \downarrow$. As we are interested in the relationship with the spin voltage $\Delta P_{s}=\Delta P^{\uparrow}-\Delta P^{\downarrow}$, we subtract the two Eqs. (C14) and finally find

$$
\frac{1}{\chi_{s}} \Delta m=\Delta P_{s}+\frac{D_{0}^{\uparrow \prime}}{D_{0}^{\uparrow}}\left(\mu_{0}\right) \Delta A^{\uparrow}-\frac{D_{0}^{\downarrow \prime}}{D_{0}^{\downarrow}}\left(\mu_{0}\right) \Delta A^{\downarrow},
$$

where the Pauli spin susceptibility $\chi_{s}>0$ is given by

$$
\frac{1}{\chi_{s}}=\frac{1}{2 D_{0}^{\uparrow}\left(\mu_{0}\right)}+\frac{1}{2 D_{0}^{\downarrow}\left(\mu_{0}\right)}-I .
$$

We, thus, express $\Delta m$ by the moments $\Delta P_{s}$ and $\Delta A^{\sigma}$. Note that, in standard thermodynamics with state variables $\mu_{s}=\mu^{\uparrow}-\mu^{\downarrow}$ and one temperature $T$, Eq. (C15) corresponds to

$$
\Delta m=\frac{\partial m}{\partial \mu_{s}} \Delta \mu_{s}+\frac{\partial m}{\partial T} \Delta T .
$$

From Eq. (C15), we can substitute $\Delta P_{s}$ into Eq. (C10) and obtain the equation of motion for $\Delta m$,

$$
\left(\partial_{t}+\Gamma_{\mathrm{es}}\right) \Delta m=\Delta F
$$

where $\Gamma_{\mathrm{es}}=2 g_{\mathrm{sf}}\left(\mu_{0}\right) / \chi_{\mathrm{s}}$ and

$$
\begin{aligned}
\Delta F= & 2 g_{\mathrm{sf}}\left(\frac{D_{0}^{\uparrow \prime}}{D_{0}^{\uparrow}}-\frac{g_{\mathrm{sf}}^{\prime}}{g_{\mathrm{sf}}}\right)\left(\mu_{0}\right) \Delta A^{\uparrow} \\
& -2 g_{\mathrm{sf}}\left(\frac{D_{0}^{\downarrow \prime}}{D_{0}^{\downarrow}}-\frac{g_{\mathrm{sf}}^{\prime}}{g_{\mathrm{sf}}}\right)\left(\mu_{0}\right) \Delta A^{\downarrow}
\end{aligned}
$$


is the force that drives the magnetization change. It is proportional to a weighted average of the generalized excess electron temperatures. The solution of Eq. (C18) is given by the convolution

$\Delta m(t)=\left(H_{\mathrm{es}} * \Delta F\right)(t)=\int d \tau H_{\mathrm{es}}(t-\tau) \Delta F(\tau)$

of $\Delta F$ with a response function $H_{\mathrm{es}}(t)=\Theta(t) \mathrm{e}^{-\Gamma_{\mathrm{es}} t}$, where $\Theta(t)$ is the Heaviside step function. The time constant $\Gamma_{\mathrm{es}}^{-1}$ quantifies how quickly the magnetization adapts to a change in the squared generalized temperatures of spin-up and spindown electrons.

\section{Energy transfer to phonons}

In the framework of the Stoner model, the energy of the electrons is given by $E_{e}=\sum_{\sigma} \int d \epsilon\left(\epsilon-\mu_{0}\right) D^{\sigma} n^{\sigma}+$ $\mathrm{Im}^{2} / 4$. It is modified by excitation by the laser-pulse and electron-phonon interactions through changes $\Delta n^{\sigma}$, the sample magnetic moment $m$, and, thus, the density $D^{\sigma}$ of states. By a consideration similar to Eq. (C13), one can show that the latter two processes cancel to linear order in the $\Delta n^{\sigma}$, and one obtains

$$
\Delta E_{e}=\sum_{\sigma} C_{e}^{\sigma} \Delta \tilde{T}^{\sigma}
$$

for the electron excess energy. Here, $C_{e}^{\sigma}=\left(\pi^{2} k_{B}^{2} / 3\right) \times$ $T_{0} D_{0}^{\sigma}\left(\mu_{0}\right), \Delta \tilde{T}^{\sigma} \propto \Delta A^{\sigma}$ [Eq. (C8)], and $C_{e}=C_{e}^{\uparrow}+C_{e}^{\downarrow}$ is the heat capacity of the electrons. Note that $\Delta E_{e}$ is the total pump-induced excess energy of the electrons, which captures both their orbital and spin degrees of freedom.

Equation (C21) underscores the interpretation of $T_{0}+\Delta \tilde{T}^{\sigma}$ as generalized temperature [Eq. (C8)] and allows us to rephrase the interpretation of Eq. (C18): Once spin-up and spin-down electrons are equilibrated $\left(\Delta \tilde{T}^{\uparrow}=\Delta \tilde{T}^{\downarrow}\right), \quad \Delta F$ is directly proportional to the excess energy of all electrons, independent of the precise shape of the electron distributions. We emphasize that Eq. (C21) is valid in the limit that the relevant effects scale linearly with the occupation changes $\Delta n^{\sigma}$. Beyond this regime, at higher pump fluences, explicit spin terms containing the magnetization would show up in Eq. (C21).

After excitation by the pump pulse, the total excess electron energy $\Delta E_{e}$ decays due to electron-phonon (ep) interactions. To determine the dynamics of $\Delta E_{e}$, we neglect spin flips and use the relationship [48]

$$
\begin{aligned}
\left.\Delta \dot{E}_{e}\right|_{\mathrm{ep}} & \propto \sum_{\sigma} \int d \delta\left(\alpha^{2} F\right)^{\sigma}(\delta) \int d \epsilon\left\{\left[n^{\sigma}(\epsilon)\right.\right. \\
& \left.\left.-n^{\sigma}(\epsilon+\delta)\right] p(\delta)-\left[1-n^{\sigma}(\epsilon)\right] n^{\sigma}(\epsilon+\delta)\right\}
\end{aligned}
$$

Here, $\left(\alpha^{2} F\right)^{\sigma}(\delta)$ denotes the Eliashberg function that describes the coupling of phonons of energy $\delta$ with two electronic states of the same spin $\sigma$ and energy $\epsilon$ and $\epsilon+\delta$. The occupation of phonons with energy $\delta$ is given by $p(\delta)$. Note that the term under the $\epsilon$ integral becomes zero for all $\delta$ and $\epsilon$ if $n^{\sigma}$ is a Fermi-Dirac distribution and $p$ is a BoseEinstein distribution with the same temperature.

Following Ref. [7], we linearize Eq. (C22) with respect to $\Delta n^{\sigma}=n^{\sigma}-n_{0}$ and $\Delta p=p-p_{0}$ to obtain

$$
\begin{aligned}
\left.\Delta \dot{E}_{e}\right|_{\mathrm{ep}} & \propto \sum_{\sigma} \int d \delta\left(\alpha^{2} F\right)^{\sigma}(\delta) \delta \Delta p(\delta)-\sum_{\sigma} \int d \epsilon \Delta n^{\sigma}(\epsilon) \\
& \times \int d \delta\left(\alpha^{2} F\right)^{\sigma}\left[1-n_{0}(\epsilon-\delta)-n_{0}(\epsilon+\delta)\right] . \quad(\mathrm{C} 23)
\end{aligned}
$$

Because the weight factor of $\Delta n^{\sigma}(\epsilon)$ in Eq. (C23) is sufficiently smooth, it is legitimate to apply the moment expansion of Eq. (C7), resulting in

$$
\begin{aligned}
\left.\Delta \dot{E}_{e}\right|_{\mathrm{ep}} & \propto \sum_{\sigma} \int d \delta\left(\alpha^{2} F\right)^{\sigma}(\delta) \delta \Delta p(\delta) \\
& -\sum_{\sigma} \Delta A^{\sigma} \int d \delta\left(\alpha^{2} F\right)^{\sigma}(\delta)\left[-2 n_{0}^{\prime}\left(\mu_{0}-\delta\right)\right] .
\end{aligned}
$$

Note that the first integral approximately scales with the pump-induced phonon excess energy, because $\left(\alpha^{2} F\right)^{\sigma}(\delta)$ is approximately proportional to the phonon density of states [48]. Owing to Eqs. (C8) and (C21), the second integral approximately scales with the excess energy of the $\sigma$ electrons. The generalized chemical potential does not show up in Eq. (C24), as the weight factor of $\Delta n^{\sigma}(\epsilon)$ in Eq. (C23) is antisymmetric with respect to $\epsilon-\mu_{0}$.

When we finally assume that the phonon distribution $p_{0}+\Delta p$ is thermal and obeys a Bose-Einstein distribution at temperature $T_{0}+\Delta T_{p}$, Eq. (C24) along with Eqs. (C8) and $(\mathrm{C} 21)$ leads to the familiar result

$$
\left.C_{e} \Delta \tilde{T}_{e}\right|_{\mathrm{ep}}=-G_{\mathrm{ep}} \cdot\left(\Delta \tilde{T}_{e}-\Delta T_{p}\right) .
$$

Here, $C_{e}=C_{e}^{\uparrow}+C_{e}^{\downarrow}$ is the total electronic heat capacity, and the coupling strength $G_{\mathrm{ep}}$ is proportional to $\sum_{\sigma} \int d \delta\left(\alpha^{2} F\right)^{\sigma}(\delta)\left[-2 n_{0}^{\prime}\left(\mu_{0}-\delta\right)\right]$. In the last step to Eq. (C25), we took advantage of the fact that $\left.\Delta \dot{E}_{e}\right|_{\text {ep }}=0$ when $\Delta \tilde{T}_{e}=\Delta T_{p}$. We emphasize that the electron-phonon energy-transfer rate is given by the difference between the generalized temperature of the $\sigma$ electrons and the thermal phonons, independent of the precise shape of the possibly nonthermal electron distribution.

Energy conservation implies that $C_{e} \Delta \tilde{T}_{e}+C_{p} \Delta T_{p}=$ $C_{e} \Delta \tilde{T}_{e 0}$ after excitation, where $C_{p}$ is the phononic heat capacity and $\Delta \tilde{T}_{e 0}$ is the increase of the electron temperature directly after excitation by the $\delta$-like pump pulse at $t=0$. Substitution of $\Delta T_{p}$ into Eq. (C25) yields the solution 


$$
\Delta \tilde{T}_{e}(t)=\Theta(t) \Delta \tilde{T}_{e 0}\left[(1-R) e^{-\Gamma_{\mathrm{ep}} t}+R\right],
$$

where $R=C_{e} /\left(C_{p}+C_{e}\right)$ and $\Gamma_{\mathrm{ep}}=G_{\mathrm{ep}} / C_{e}$.

\section{Distribution function after pump absorption}

The change $\Delta n(\epsilon)$ in the electron occupation number directly after absorption of a pump pulse with photon energy $\hbar \omega_{p}$ is proportional to the strength of transitions from states with energy $\epsilon \pm \hbar \omega_{p}$, that is,

$$
\begin{aligned}
\Delta n(\epsilon)= & C(\epsilon)\left\{\left[n_{0}\left(\epsilon-\hbar \omega_{p}\right)-n_{0}(\epsilon)\right]\right. \\
& \left.+\left[n_{0}\left(\epsilon+\hbar \omega_{p}\right)-n_{0}(\epsilon)\right]\right\} .
\end{aligned}
$$

Here, $n_{0}(\epsilon)$ is a Fermi-Dirac distribution with temperature $T=T_{0}$ and chemical potential (Fermi level) $\mu_{0}$. The prefactor $C(\epsilon)$ captures matrix elements, densities of states, and the pump-pulse energy and is assumed to be constant over the relevant electron energies. The absorbed pump energy is given by

$$
\begin{aligned}
\Delta W_{\mathrm{abs}} & =\Delta E_{e}=\int d \epsilon\left(\epsilon-\mu_{0}\right) D_{0}(\epsilon) \Delta n(\epsilon) \\
& \approx D_{0}\left(\mu_{0}\right) \int d \epsilon\left(\epsilon-\mu_{0}\right) \Delta n(\epsilon)
\end{aligned}
$$

where $D_{0}=D_{0}^{\uparrow}+D_{0}^{\downarrow}$ is the electronic density of states before arrival of the pump pulse. In the last step of Eq. (C28), we apply the moment expansion of Eq. (C7). The density of states can be obtained from the known molar electronic specific heat $C_{e} / N=\gamma T_{0}$ of Fe, where $N$ is the number of $\mathrm{Fe}$ atoms and $\gamma \approx 5 \mathrm{~mJ} \mathrm{~mol}^{-1} \mathrm{~K}^{-2}$ [36], and $C_{e}=\left(\pi^{2} k_{B}^{2} / 3\right) T_{0} D_{0}\left(\mu_{0}\right) \quad$ [see Eq. $\left.\quad(\mathrm{C} 21)\right]$, yielding $D_{0}\left(\mu_{0}\right) / N=3 \gamma / \pi^{2} k_{B}^{2} \approx 2.1 \mathrm{eV}^{-1}$ per Fe atom.

On the other hand, the absorbed pump energy per $\mathrm{Fe}$ atom can be calculated as $\Delta W_{\text {abs }} / N=F_{\text {abs }} v / d$, where $F_{\text {abs }}$ is the absorbed pump fluence, $d=4 \mathrm{~nm}$ is the Fe film thickness, and $v=V / N=0.012 \mathrm{~nm}^{3}$ is the volume per Fe atom. Combining these considerations with Eqs. (C27) and (C28) allows us to determine $C$ and yields the change in the electron occupation number directly after pump absorption:

$$
\Delta n(\epsilon)=\frac{F_{\mathrm{abs}} v / d}{D_{0}\left(\mu_{0}\right) / N} \frac{n_{0}\left(\epsilon-\hbar \omega_{p}\right)-2 n_{0}(\epsilon)+n_{0}\left(\epsilon+\hbar \omega_{p}\right)}{\int d \epsilon\left(\epsilon-\mu_{0}\right)\left[n_{0}\left(\epsilon-\hbar \omega_{p}\right)-2 n_{0}(\epsilon)+n_{0}\left(\epsilon+\hbar \omega_{p}\right)\right]} .
$$

Figure 1(b) of the main text shows calculations of $\Delta n(\epsilon)$ for optical $(3.1 \mathrm{eV})$ and $\mathrm{THz}(4.1 \mathrm{meV})$ photons for an absorbed fluence of $F_{\text {abs }}=0.01 \mathrm{~mJ} \mathrm{~cm}^{-2}$. This value is slightly smaller than the absorbed THz-pump fluence in the experiment $\left(0.017 \mathrm{~mJ} \mathrm{~cm}^{-2}\right)$, since the $\mathrm{THz}$ pulse duration is comparable to the electron-phonon thermalization time. Fitting the resulting $\Delta n(\epsilon)$ by a Fermi function (temperature $T_{0}+\Delta T_{e}$ and chemical potential $\left.\mu_{0}\right)$ minus $n_{0}(\epsilon)$ yields a change of $\Delta T_{e}=140 \mathrm{~K}$ in the electron temperature [see Fig. 1(b)]. For comparison, using the electron specific heat [49], we obtain $\Delta T_{e}=\Delta W_{\text {abs }} / C_{e}=120 \mathrm{~K}$, which is in good agreement with the fit approach.

[1] E. Y. Vedmedenko, R. K. Kawakami, D. D. Sheka, P. Gambardella, A. Kirilyuk, A. Hirohata, C. Binek, O. Chubykalo-Fesenko, S. Sanvito, B. J. Kirby, J. Grollier, K. Everschor-Sitte, T. Kampfrath, C. Y. You, and A. Berger, The 2020 Magnetism Roadmap, J. Phys. D53, 453001 (2020).

[2] A. Kirilyuk, A. V. Kimel, and T. Rasing, Laser-Induced Magnetization Dynamics and Reversal in Ferrimagnetic Alloys, Rep. Prog. Phys. 76, 026501 (2013).

[3] E. Beaurepaire, J. C. Merle, A. Daunois, and J. Y. Bigot, Ultrafast Spin Dynamics in Ferromagnetic Nickel, Phys. Rev. Lett. 76, 4250 (1996).

[4] E. Carpene, E. Mancini, C. Dallera, M. Brenna, E. Puppin, and S. De Silvestri, Dynamics of Electron-Magnon
Interaction and Ultrafast Demagnetization in Thin Iron Films, Phys. Rev. B 78, 174422 (2008).

[5] B. Koopmans, G. Malinowski, F. Dalla Longa, D. Steiauf, M. Fähnle, T. Roth, M. Cinchetti, and M. Aeschlimann, Explaining the Paradoxical Diversity of Ultrafast Laser-Induced Demagnetization, Nat. Mater. 9, 259 (2010).

[6] K. Bühlmann, G. Saerens, A. Vaterlaus, and Y. Acremann, Detection of Femtosecond Spin Voltage Pulses in a Thin Iron Film, Struct. Dyn. 7, 065101 (2020).

[7] R. Rouzegar, L. Brandt, L. Nadvornik, D. A. Reiss, A. L. Chekhov, O. Gueckstock, C. In, M. Wolf, T. S. Seifert, P. W. Brouwer, G. Woltersdorf, and T. Kampfrath, Laser-Induced Terahertz Spin Transport in Magnetic Nanostructures Arises from the Same Force as Ultrafast Demagnetization, arXiv:2103.11710.

[8] T. Lichtenberg, M. Beens, M. H. Jansen, R. A. Duine, and B. Koopmans, Probing Optical Spin-Currents Using $\mathrm{THz}$ Spin-Waves in Noncollinear Magnetic Bilayers, arXiv:2103 .06029 .

[9] B. Y. Mueller and B. Rethfeld, Thermodynamic $\mu T$ Model of Ultrafast Magnetization Dynamics, Phys. Rev. B 90, 144420 (2014).

[10] I. Razdolski, A. Alekhin, N. Ilin, J. P. Meyburg, V. Roddatis, D. Diesing, U. Bovensiepen, and A. Melnikov, Nanoscale Interface Confinement of Ultrafast Spin Transfer Torque Driving Non-Uniform Spin Dynamics, Nat. Commun. 8, 15007 (2017).

[11] T. Seifert et al., Efficient Metallic Spintronic Emitters of Ultrabroadband Terahertz, Radiation, Nat. Photonics 10, 483 (2016). 
[12] E. T. Papaioannou, G. Torosyan, S. Keller, L. Scheuer, M. Battiato, V. K. Mag-Usara, J. L'Huillier, M. Tani, and R. Beigang, Efficient Terahertz Generation Using Fe/Pt Spintronic Emitters Pumped at Different Wavelengths, IEEE Trans. Magn. 54, 9100205 (2018).

[13] R. I. Herapath, S. M. Hornett, T. S. Seifert, G. Jakob, M. Kläui, J. Bertolotti, T. Kampfrath, and E. Hendry, Impact of Pump Wavelength on Terahertz Emission of a CavityEnhanced Spintronic Trilayer, Appl. Phys. Lett. 114, 041107 (2019).

[14] U. Atxitia, O. Chubykalo-Fesenko, J. Walowski, A. Mann, and M. Münzenberg, Evidence for Thermal Mechanisms in Laser-Induced Femtosecond Spin Dynamics, Phys. Rev. B 81, 174401 (2010).

[15] K. Carva, M. Battiato, and P. M. Oppeneer, Ab Initio Investigation of the Elliott-Yafet Electron-Phonon Mechanism in Laser-Induced Ultrafast Demagnetization, Phys. Rev. Lett. 107, 207201 (2011).

[16] M. S. Si and G. P. Zhang, Hot Spin Spots in the LaserInduced Demagnetization, AIP Adv. 2, 012158 (2012).

[17] A. Eschenlohr, M. Battiato, P. Maldonado, N. Pontius, T. Kachel, K. Holldack, R. Mitzner, A. Föhlisch, P. M. Oppeneer, and C. Stamm, Ultrafast Spin Transport as Key to Femtosecond Demagnetization, Nat. Mater. 12, 332 (2013).

[18] E. Turgut, D. Zusin, D. Legut, K. Carva, R. Knut, J. M. Shaw, C. Chen, Z. Tao, H. T. Nembach, T. J. Silva, S. Mathias, M. Aeschlimann, P. M. Oppeneer, H. C. Kapteyn, M. M. Murnane, and P. Grychtol, Stoner versus Heisenberg: Ultrafast Exchange Reduction and Magnon Generation during Laser-Induced Demagnetization, Phys. Rev. B 94, 220408(R) (2016).

[19] K. Bühlmann, R. Gort, G. Salvatella, S. Däster, A. Fognini, T. Bähler, C. Dornes, C. A. F. Vaz, A. Vaterlaus, and Y. Acremann, Ultrafast Demagnetization in Iron: Separating Effects by Their Nonlinearity, Struct. Dyn. 5, 044502 (2018).

[20] U. Bovensiepen, Coherent and Incoherent Excitations of the Gd(0001) Surface on Ultrafast Timescales, J. Phys. Condens. Matter 19, 083201 (2007).

[21] M. Lisowski, P. A. Loukakos, U. Bovensiepen, J. Stähler, C. Gahl, and M. Wolf, Ultra-Fast Dynamics of Electron Thermalization, Cooling and Transport Effects in Ru (001), Appl. Phys. A 78, 165 (2004).

[22] T. S. Seifert, S. Jaiswal, J. Barker, S. T. Weber, I. Razdolski, J. Cramer, O. Gueckstock, S. F. Maehrlein, L. Nadvornik, S. Watanabe, C. Ciccarelli, A. Melnikov, G. Jakob, M. Münzenberg, S. T. B. Goennenwein, G. Woltersdorf, B. Rethfeld, P. W. Brouwer, M. Wolf, M. Kläui, and T. Kampfrath, Femtosecond Formation Dynamics of the Spin Seebeck Effect Revealed by Terahertz Spectroscopy, Nat. Commun. 9, 2899 (2018).

[23] K. Carva, M. Battiato, D. Legut, and P. M. Oppeneer, Ab Initio Theory of Electron-Phonon Mediated Ultrafast Spin Relaxation of Laser-Excited Hot Electrons in Transition-Metal Ferromagnets, Phys. Rev. B 87, 184425 (2013).

[24] J. H. Shim, A. A. Syed, J. Il Kim, H. G. Piao, S. H. Lee, S. Y. Park, Y. S. Choi, K. M. Lee, H. J. Kim, J. R. Jeong, J. Il Hong, D. E. Kim, and D. H. Kim, Role of Non-Thermal
Electrons in Ultrafast Spin Dynamics of Ferromagnetic Multilayer, Sci. Rep. 10, 6355 (2020).

[25] T. Roth, A. J. Schellekens, S. Alebrand, O. Schmitt, D. Steil, B. Koopmans, M. Cinchetti, and M. Aeschlimann, Temperature Dependence of Laser-Induced Demagnetization in Ni: A Key for Identifying the Underlying Mechanism, Phys. Rev. X 2, 021006 (2012).

[26] B. Y. Mueller, T. Roth, M. Cinchetti, M. Aeschlimann, and B. Rethfeld, Driving Force of Ultrafast Magnetization Dynamics, New J. Phys. 13, 123010 (2011).

[27] A. Zagdoud, M. Vomir, M. Albrecht, M. Barthelemy, and J.-Y. Bigot, in Proceedings of the International Conference on Ultrafast Phenomena (2010) (The Optical Society, Washington, DC, 2013), paper TuE49, 10.1364/UP.2010.TuE49.

[28] V. Cardin, T. Balciunas, K. Légaré, A. Baltuska, H. Ibrahim, E. Jal, B. Vodungbo, N. Jaouen, C. Varin, J. Lüning, and F. Légaré, Wavelength Scaling of Ultrafast Demagnetization in Co/Pt Multilayers, Phys. Rev. B 101, 054430 (2020).

[29] S. Bonetti, M. C. Hoffmann, M. J. Sher, Z. Chen, S. H. Yang, M. G. Samant, S. S. P. Parkin, and H. A. Dürr, THzDriven Ultrafast Spin-Lattice Scattering in Amorphous Metallic Ferromagnets, Phys. Rev. Lett. 117, 087205 (2016).

[30] M. Shalaby, A. Donges, K. Carva, R. Allenspach, P. M. Oppeneer, U. Nowak, and C. P. Hauri, Coherent and Incoherent Ultrafast Magnetization Dynamics in 3d Ferromagnets Driven by Extreme Terahertz Fields, Phys. Rev. B 98, 014405 (2018).

[31] R. B. Wilson, Y. Yang, J. Gorchon, C. H. Lambert, S. Salahuddin, and J. Bokor, Electric Current Induced Ultrafast Demagnetization, Phys. Rev. B 96, 045105 (2017).

[32] E. A. Mashkovich, K. A. Grishunin, H. Munekata, and A. V. Kimel, Ultrafast Demagnetization of Ferromagnetic Semiconductor InMnAs by Dual Terahertz and Infrared Excitations, Appl. Phys. Lett. 117, 122406 (2020).

[33] M. Hudl, M. d'Aquino, M. Pancaldi, S.-H. Yang, M. G. Samant, S. S. P. Parkin, H. A. Dürr, C. Serpico, M. C. Hoffmann, and S. Bonetti, Nonlinear Magnetization Dynamics Driven by Strong Terahertz Fields, Phys. Rev. Lett. 123, 197204 (2019).

[34] J. Hohlfeld, S. S. Wellershoff, J. Güdde, U. Conrad, V. Jähnke, and E. Matthias, Electron and Lattice Dynamics Following Optical Excitation of Metals, Chem. Phys. 251, 237 (2000).

[35] See Supplemental Material at http://link.aps.org/ supplemental/10.1103/PhysRevX.11.041055 for details on experimental setup, raw data, deconvolution procedure, fluence dependencies of the signals, $\mathrm{THz}$ transmission spectroscopy, nonmagnetic dynamics, and extraction of pure magnetization dynamics.

[36] M. A. Ordal, R. J. Bell, R. W. Alexander, L. A. Newquist, and M. R. Querry, Optical Properties of Al, Fe, Ti, Ta, W, and Mo at Submillimeter Wavelengths, Appl. Opt. 27, 1203 (1988).

[37] H. Hirori, A. Doi, F. Blanchard, and K. Tanaka, SingleCycle Terahertz Pulses with Amplitudes Exceeding $1 \mathrm{MV} / \mathrm{Cm}$ Generated by Optical Rectification in LiNbO3, Appl. Phys. Lett. 98, 091106 (2011). 
[38] M. Sajadi, M. Wolf, and T. Kampfrath, Terahertz-FieldInduced Optical Birefringence in Common Window and Substrate Materials, Opt. Express 23, 28985 (2015).

[39] S. Maehrlein, A. Paarmann, M. Wolf, and T. Kampfrath, Terahertz Sum-Frequency Excitation of a Raman-Active Phonon, Phys. Rev. Lett. 119, 127402 (2017).

[40] S. F. Maehrlein, I. Radu, P. Maldonado, A. Paarmann, M. Gensch, A. M. Kalashnikova, R. V. Pisarev, M. Wolf, P. M. Oppeneer, J. Barker, and T. Kampfrath, Dissecting SpinPhonon Equilibration in Ferrimagnetic Insulators by Ultrafast Lattice Excitation, Sci. Adv. 4, eaar5164 (2018).

[41] T. Kampfrath, R. G. Ulbrich, F. Leuenberger, M. Münzenberg, B. Sass, and W. Felsch, Ultrafast MagnetoOptical Response of Iron Thin Films, Phys. Rev. B 65, 104429 (2002).

[42] B. Koopmans, M. Van Kampen, and W. J. M. De Jonge, Experimental Access to Femtosecond Spin Dynamics, J. Phys. Condens. Matter 15, S723 (2003).

[43] I. Razdolski, A. Alekhin, U. Martens, D. Bü Rstel, D. Diesing, M. Münzenberg, U. Bovensiepen, and A. Melnikov, Analysis of the Time-Resolved Magneto-Optical Kerr Effect for Ultrafast Magnetization Dynamics in Ferromagnetic Thin Films, J. Phys. Condens. Matter 29, 174002 (2017).

[44] E. Carpene, H. Hedayat, F. Boschini, and C. Dallera, Ultrafast Demagnetization of Metals: Collapsed Exchange versus Collective Excitations, Phys. Rev. B 91, 174414 (2015).

[45] C. Vicario, C. Ruchert, F. Ardana-Lamas, P. M. Derlet, B. Tudu, J. Luning, and C. P. Hauri, Off-Resonant Magnetization Dynamics Phase-Locked to an Intense Phase-Stable Terahertz Transient, Nat. Photonics 7, 720 (2013).

[46] S. Ogawa, H. Nagano, H. Petek, and A. P. Heberle, Optical Dephasing in Cu(111) Measured by Interferometric TwoPhoton Time-Resolved Photoemission, Phys. Rev. Lett. 78, 1339 (1997).

[47] B. Y. Mueller, A. Baral, S. Vollmar, M. Cinchetti, M. Aeschlimann, H. C. Schneider, and B. Rethfeld, Feedback Effect during Ultrafast Demagnetization Dynamics in Ferromagnets, Phys. Rev. Lett. 111, 167204 (2013).

[48] P. B. Allen, Theory of Thermal Relaxation of Electrons in Metals, Phys. Rev. Lett. 59, 1460 (1987).

[49] Q. Chen and B. Sundman, Modeling of Thermodynamic Properties for Bcc, Fcc, Liquid, and Amorphous Iron, J. Phase Equilib. 22, 631 (2001).

[50] H. S. Rhie, H. A. Dürr, and W. Eberhardt, Femtosecond Electron and Spin Dynamics in [Formula Presented] Films, Phys. Rev. Lett. 90, 247201 (2003).
[51] P. Tengdin, W. You, C. Chen, X. Shi, D. Zusin, Y. Zhang, C. Gentry, A. Blonsky, M. Keller, P. M. Oppeneer, H. C. Kapteyn, Z. Tao, and M. M. Murnane, Critical Behavior within 20 Fs Drives the Out-of-Equilibrium Laser-Induced Magnetic Phase Transition in Nickel, Sci. Adv. 4, eaap9744 (2018).

[52] R. Gort, K. Bühlmann, S. Däster, G. Salvatella, N. Hartmann, Y. Zemp, S. Holenstein, C. Stieger, A. Fognini, T. U. Michlmayr, T. Bähler, A. Vaterlaus, and Y. Acremann, Early Stages of Ultrafast Spin Dynamics in a $3 d$ Ferromagnet, Phys. Rev. Lett. 121, 087206 (2018).

[53] W. S. Fann, R. Storz, H. W. K. Tom, and J. Bokor, Electron Thermalization in Gold, Phys. Rev. B 46, 13592 (1992).

[54] A. K. Zvezdin and V. A. Kotov, Modern Magnetooptics and Magnetooptical Materials (Taylor \& Francis, New York, 1997).

[55] R. Adam, G. Chen, D. E. Bürgler, T. Shou, I. Komissarov, S. Heidtfeld, H. Hardtdegen, M. Mikulics, C. M. Schneider, and R. Sobolewski, Magnetically and Optically Tunable Terahertz Radiation from Ta/NiFe/Pt Spintronic Nanolayers Generated by Femtosecond Laser Pulses, Appl. Phys. Lett. 114, 212405 (2019).

[56] A. Grechnev, I. Di Marco, M. I. Katsnelson, A. I. Lichtenstein, J. Wills, and O. Eriksson, Theory of Bulk and Surface Quasiparticle Spectra for Fe, Co, and Ni, Phys. Rev. B 76, 035107 (2007).

[57] O. Gueckstock, L. Nádvorník, M. Gradhand, T. S. Seifert, G. Bierhance, R. Rouzegar, M. Wolf, M. Vafaee, J. Cramer, M. A. Syskaki, G. Woltersdorf, I. Mertig, G. Jakob, M. Kläui, and T. Kampfrath, Terahertz. Spin-to-Charge Conversion by Interfacial Skew Scattering in Metallic Bilayers, Adv. Mater. 33, 2006281 (2021).

[58] L. Nadvorník, M. Borchert, L. Brandt, R. Schlitz, K. A. de Mare, K. Výborný, I. Mertig, G. Jakob, M. Kläui, S. T. B. Goennenwein, M. Wolf, G. Woltersdorf, and T. Kampfrath, Broadband Terahertz Probes of Anisotropic Magnetoresistance Disentangle Extrinsic and Intrinsic Contributions, Phys. Rev. X 11, 021030 (2021).

[59] C. Koike, H. Hasegawa, N. Asada, and T. Komatuzaki, Optical Constants of Fine Particles for the Infrared Region, Mon. Not. R. Astron. Soc. 239, 127 (1989).

[60] D. A. Naylor and M. K. Tahic, Apodizing Functions for Fourier Transform Spectroscopy, J. Opt. Soc. Am. A 24, 3644 (2007). 